\title{
First-principle based multi-channel integrated modelling in support to the design of the Divertor Tokamak Test facility
}

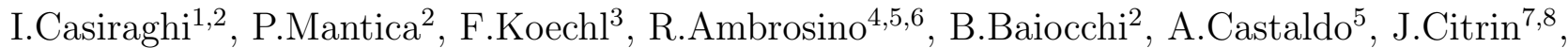 \\ M.Dicorato ${ }^{1}$, L.Frassinetti ${ }^{9}$, A.Mariani ${ }^{1,2}$, P.Vincenzi ${ }^{10}$, P.Agostinetti ${ }^{10}$, L.Aucone ${ }^{11}$, L.Balbinot ${ }^{10,12}$, \\ S.Ceccuzzi $^{6,13}$, L.Figini ${ }^{2}$, G.Granucci ${ }^{2}$, P.Innocente ${ }^{10}$, T.Johnson ${ }^{9}$, H.Nyström ${ }^{9}$, M.Valisa ${ }^{10}$ \\ ${ }^{1}$ Dipartimento di Fisica "G. Occhialini”, Università di Milano-Bicocca, Milano, Italy \\ 2 Istituto per la Scienza e Tecnologia dei Plasmi, CNR, Milano, Italy \\ ${ }^{3} \mathrm{CCFE}$, Culham Science Centre, Abingdon, UK \\ ${ }^{4}$ Università degli Studi di Napoli Federico II, Napoli, Italy \\ ${ }^{5}$ Consorzio CREATE, Napoli, Italy \\ ${ }^{6}$ DTT S.C. a r.l., Frascati, Italy, \\ ${ }^{7}$ DIFFER - Dutch Institute for Fundamental Energy Research, Eindhoven, Netherlands \\ ${ }^{8}$ Science and Technology of Nuclear Fusion Group, Eindhoven University of Technology, Eindhoven, Netherlands \\ ${ }^{9}$ Fusion Plasma Physics, ECSS, KTH Royal Institute of Technology, Stockholm, Sweden \\ ${ }^{10}$ Consorzio RFX, Padova, Italy \\ ${ }^{11}$ Politecnico di Milano, Milano, Italy \\ ${ }^{12}$ Università degli Studi di Padova, Padova, Italy \\ ${ }^{13}$ ENEA C.R.Frascati, Frascati, Italy
}

July 25, 2021

\begin{abstract}
An intensive integrated modelling work of main scenarios of the new tokamak DTT (Divertor Tokamak Test facility) with the Single Null divertor configuration has been performed using first-principle quasi-linear transport models, in support to the design of the device and to the definition of its scientific work-programme. First results of this integrated modelling work on DTT $\left(R_{0}=2.14 \mathrm{~m}, a=0.65 \mathrm{~m}\right)$ are presented here along with outcome of the gyrokinetic simulations used to validate the reduced models in the DTT range of parameters. As a result of this work, the heating mix was defined, the size of device was increased to $R_{0}=2.19 \mathrm{~m}$ and $a=0.70 \mathrm{~m}$, the use of pellets for fuelling has been advised and reference profiles for diagnostic design, estimates of neutron yields and fast particle losses have become available.
\end{abstract}

\section{Introduction}

Studying the controlled power and particle exhaust from a fusion reactor is a main research topic in the European Fusion Roadmap[1, 2].

ITER[3] (International Thermonuclear Experimental Reactor) is planned to test a conventional metal divertor operating in a plasma fully detached condition. This baseline approach to the power exhaust problem may not be suitable for extrapolation to the operating conditions of DEMO[4] (DEMOnstration power plant) and future reactors, requiring plasma facing components able to cope with huge power fluxes in the range of $10-20 \mathrm{MW} / \mathrm{m}^{2}$. Therefore, studying and developing an alternative exhaust strategy is crucial to mitigate the risk.
This is the main task of the new tokamak DTT (Divertor Tokamak Test facility) $[5,6,7]$, whose construction is starting in Frascati, Italy, with the first plasma planned for 2026. The DTT design is in advanced status but will be kept flexible with regard to the choice of the divertor until 2023, when the outcome of the work conducted under the EUROfusion PEX ad hoc group will be available to drive the best choice for the DTT divertor.

For the optimisation of the various aspects of the DTT design, it is of key importance to perform integrated modelling of the foreseen operational scenarios using first principle based transport models and state-of-art modules for heating, fuelling and magnetic equilibrium. Integrated modelling allows to predict main plasma profiles as a result of non-linear interactions between plasma, heating 


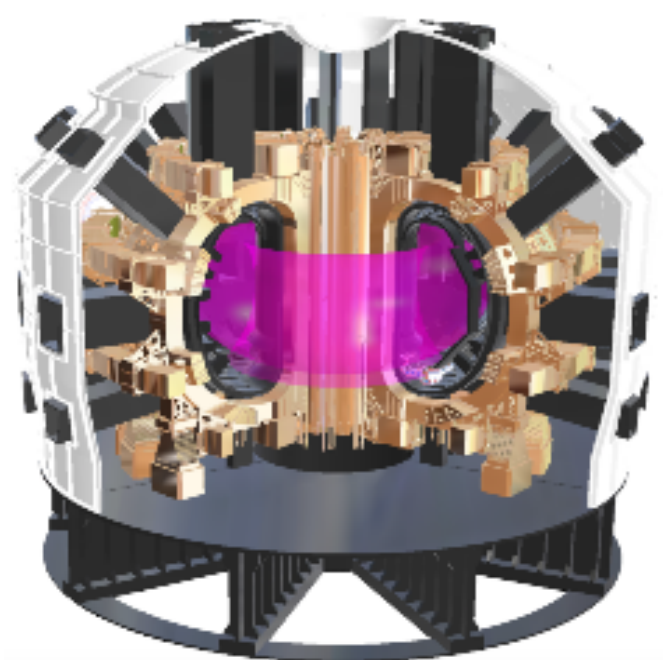

Figure 1: DTT device.

and fuelling, and impurity influxes, as well as amongst different transport channels.

This is the aim of this paper, which reports the first DTT simulations using theory-based transport models, to support the DTT design, and particularly the definition of the heating mix, the design of the neutron shields, the assessment of fast particle losses and the design of diagnostic systems, as well as to help the elaboration of a DTT scientific work-programme.

\section{The DTT project}

The new Italian tokamak DTT is a D-shaped superconducting device, whose construction is starting at the ENEA Research Center in Frascati, Italy. A drawing of the DTT device is shown in figure 1.

The characteristics of DTT were chosen to make it ITER and DEMO relevant, so that exhaust solutions could be extrapolated to a reactor-grade plasma. Bearing in mind the requirement of a strong compatibility with the operating conditions in DEMO, DTT is designed to be a bulk-edge integrated experiment with a reactor relevant bulk. Therefore, the DTT dimensionless physical parameters should be as close as possible to the ITER and DEMO ones. It is not possible to simultaneously preserve all these quantities, and hence DTT has been scaled down following the so called "weak scaling" described in [8]. Since the $P_{\mathrm{sep}} / R$ parameter, where $P_{\text {sep }}$ is the power exiting through the separatrix, is recognised as a key metric for the extent of the exhaust issue in a tokamak, geometry and auxiliary power coupled to the plasma have been chosen to guarantee a value of $P_{\mathrm{sep}} / R=15 \mathrm{MW} / \mathrm{m}$ similar to those foreseen for ITER and DEMO. So, the power at the divertor in DTT and DEMO will be comparable.
Table 1: Main parameter comparison between the DTT configuration simulated in this paper and ITER and EU DEMO future devices.[10, 11, 12, 13]

\begin{tabular}{cccc}
\hline & DTT & ITER & EU DEMO \\
\hline $\mathrm{R}[\mathrm{m}]$ & 2.14 & 6.2 & 9.1 \\
\hline $\mathrm{a}[\mathrm{m}]$ & 0.65 & 2.0 & 2.93 \\
\hline $\mathrm{A}$ & 3.3 & 3.1 & 3.1 \\
\hline$I_{p l}[\mathrm{MA}]$ & 5.5 & 15 & 19.6 \\
\hline$B_{t}[\mathrm{~T}]$ & 6 & 5.3 & 5.7 \\
\hline$P_{\text {tot }}[\mathrm{MW}]$ & 45 & 150 & 460 \\
\hline$P_{\text {sep }}[\mathrm{MW}]$ & 32 & 87 & 154 \\
\hline$P_{\text {sep }} / R[\mathrm{MW} / \mathrm{m}]$ & 15 & 14 & 17 \\
\hline$\lambda_{q}[\mathrm{~mm}]$ & 0.7 & 0.9 & 1.0 \\
\hline $\mathrm{Pulse} \mathrm{length}[\mathrm{s}]$ & 100 & 400 & 7600 \\
\hline$\beta_{N}[\%]$ & 1.6 & 1.6 & 2.6 \\
\hline$\nu_{e}^{*}$ at $r / a=0.5\left[10^{-2}\right]$ & 1.1 & 1.1 & 0.5 \\
\hline$\rho^{*}\left[10^{-3}\right]$ & 3.3 & 2.0 & 1.5 \\
\hline$\langle n\rangle\left[10^{20} / \mathrm{m}^{3}\right]$ & 1.8 & 1.0 & 0.9 \\
\hline$\left\langle T_{e}\right\rangle[\mathrm{keV}]$ & 6.7 & 8.5 & 13 \\
\hline$\tau_{E}[\mathrm{~s}]$ & 0.4 & 3.6 & 4.2 \\
\hline
\end{tabular}

Table 1 shows rough indications for both dimensional and dimensionless parameters of the DTT configuration simulated in this paper compared to ITER and EU DEMO.

The collisionality has been calculated as $\nu_{e}^{*}=6.92 \times$ $10^{18}\left(q R_{0} n_{e} Z_{\text {eff }} \ln \Lambda_{e}\right)\left(T_{e}^{2} \varepsilon^{3 / 2}\right)$, where $n_{e}$ is expressed in $\mathrm{m}^{-3}, T_{e}$ is expressed in $\mathrm{eV}$, and $\varepsilon$ is the inverse aspect ratio. [9]

The superconducting coils allow for pulse length up to $100 \mathrm{~s}$, with plasma current $I_{p l} \leq 5.5 \mathrm{MA}$ and with toroidal field coils able to generate an on-axis toroidal magnetic field $B_{t} \leq 6 \mathrm{~T}$ at $R=2.14 \mathrm{~m}$. DTT has an up-down symmetric geometry, major radius $R_{0}=2.19 \mathrm{~m}$, minor radius $a=0.70 \mathrm{~m}$, elongation $\kappa \leq 1.89$, and average triangularity $\langle\delta\rangle \leq 0.4$. The device size was recently increased from the previous values $R_{0}=2.14 \mathrm{~m}, a=0.65 \mathrm{~m}$. The simulations reported here refer to the $2.14 \mathrm{~m}$ device, and have contributed to the decision of its enlargement.

For the reference baseline DTT scenarios, a Greenwald density target value of $\langle n\rangle / n_{G} \sim 0.45$ (where $n_{G}$ is the Greenwald fraction defined in [14]), has been chosen in order to have a high operational flexibility, leaving open the possibility to explore in the future scenarios with higher densities.

The plasma shape parameters of the Single Null (SN) configuration are similar to those of the present European design of DEMO $\left(R_{0} / a \approx 3.1, \kappa_{95} \approx 1.55-1.8\right.$, 
$\left.\delta_{95} \approx 0.3\right)$. The technical description of the DTT vacuum vessel, first wall and baseline divertor, and magnetic system is contained in [6].

To address the particle and power exhaust problem, alternative divertor solutions and improved plasma facing materials will be developed and tested in DTT, thanks to its high flexibility in magnetic configurations and divertor choice. The various divertor solutions and technologies include Liquid Metal Divertors (LMD), based on either capillary porous systems or boxes/pools systems, and advanced divertor configurations such as Double Null (DN), Quasi-SnowFlake (QSF), and single null with Negative Triangularity (NT) scenarios. The reference configuration that we will use in this paper is the SN.

DTT will be equipped with three auxiliary heating systems: a Negative ion-based Neutral Beam Injection (NNBI) system, a $60-90 \mathrm{MHz}$ Ion Cyclotron Resonance Heating (ICRH) system, and a $170 \mathrm{GHz}$ Electron Cyclotron Resonance Heating (ECRH) system.

In order to match ITER and DEMO values of $P_{\mathrm{SEP}} / R$, where $P_{\mathrm{SEP}}$ is the power flowing through the last closed magnetic surface, a large amount of auxiliary power is needed ( $\sim 45 \mathrm{MW}$ in the full power scenario). The 3 heating systems will be progressively realised and installed on DTT.

The first experimental plasma (day-0 scenario) will be achieved using only $8 \mathrm{MW}$ from second harmonic ECRH ( 7.2 MW at the plasma) at the half field operational point (plasma current $I_{\mathrm{pl}}=2 \mathrm{MA}$ and toroidal magnetic field $B_{t}=3 \mathrm{~T}$ ). In a couple of years, the power coupled to the plasma will be increased up to $\sim 25 \mathrm{MW}$ in the phase called day-1 scenario, working at $I_{\mathrm{pl}}=4 \mathrm{MA}$ and $B_{t}=5.85 \mathrm{~T}$. The heating mix in this initial phase has been fixed: $16 \mathrm{MW}$ from ECRH $(\sim 14.4 \mathrm{MW}$ at plasma $)$, $4 \mathrm{MW}$ from ICRH ( $\sim 3 \mathrm{MW}$ at plasma), and 7.0-7.5 MW at plasma from NBI (with a neutral beam injector at $400 \mathrm{keV}$ ). Instead, the definitive power mix for the DTT full performance scenario has been rediscussed with respect to the original options proposed in [6], following the simulation results reported in this paper, and new options have been evaluated, as discussed in sect. 3.6, within the following ranges of power at plasma: $26-36 \mathrm{MW}$ of ECRH, 3-9 MW of ICRH, and 7.5-15 MW of NBI at energies between $200-600 \mathrm{keV}$.

The amount of heating power is an order of magnitude larger than typical power densities in nowadays tokamaks and foreseen in ITER. This trait, jointly with the cryogenic system needs, forces DTT to be an actively cooled device.

In addition to the main task dedicated to plasma exhaust, DTT will be highly relevant also for tokamak physics integrated studies with reactor relevant parameters. Thanks to the high plasma core performance, DTT is located in a unique operational region, at high density but low collisionality, which is unexplored by present tokamaks (e.g. $n_{e} \sim(0.6-0.8) \times 10^{20} / \mathrm{m}^{3}$ and $\nu_{e}^{*} \approx(2.9-4.3) \times 10^{-2}$ at $r / a=0.5$ in AUG high performance plasmas, $n_{e} \sim(0.7-0.9) \times 10^{20} / \mathrm{m}^{3}$ and $\nu_{e}^{*} \approx(2.2-3.9) \times 10^{-2}$ at $r / a=0.5$ in JET-ILW baseline discharges).

Therefore, DTT will support the experimental program of ITER, operating in parallel with it, and it will address high priority issues, such as ELM pacing, pellet fuelling, management and avoidance of disruptions, burning plasma energetic particle physics, and plasma control.

\section{Integrated modelling of DTT Single Null scenarios}

The integrated modelling of various DTT scenarios with SN configuration in $\mathrm{H}$-mode has been performed. These simulations solve the transport equations for heat, particle and momentum using a first principle transport model in a self-consistent magnetic equilibrium, to predict steady-state radial profiles of the electron and ion temperatures, density (both main species and impurity), toroidal rotation, and current density. The heating profiles are also calculated consistently, as well as all non-linear interactions between heating and plasma and between the different transport channels.

As described in detail in sect. 3.2, integrated runs have been primarily done using the JINTRAC[15] suite of codes and in some cases using the ASTRA[16] transport solver with a mixed ASTRA-JINTRAC approach.

\subsection{General settings}

The performed simulations of DTT Deuterium plasmas cover the region inside the separatrix. The equilibrium is calculated self-consistently during the run, keeping fixed the boundary, as described in sect. 3.3. Approximately 4 seconds of plasma evolution needs to be simulated until convergence, due to the current diffusion time.

The transport equations are solved within $\rho_{\text {tor }}=0.94$, where $\rho_{\text {tor }}:=\sqrt{\frac{\left(\Phi / \pi B_{\text {tor }}\right)}{\left(\Phi / \pi B_{\text {tor }}\right)_{\max }}}$ is the normalised effective minor radius, i.e. the normalised radius that a magnetic surface with circular section should have to enclose the same toroidal magnetic flux $\Phi$. The values at the top of the edge pedestal are used as boundary condition.

The pedestal pressure has been previously calculated by the Europed code[17] with the EPED1 model[63], which is based on two concepts. The pedestal transport is determined by turbulence driven by the kinetic ballooning modes (KBM) which sets a soft boundary for the gradient. This is implemented in the code via the simple expression width $=0.076 \times \sqrt{\beta_{\text {pol }}^{\text {ped }}}$, which provides one constraint that determines the gradient. However, once the pedestal reaches the KBM constraint, the pedestal height can still increase via the widening of the pedestal width. The widening continues till the peeling-ballooning (PB) 
modes are destabilised and an ELM is triggered. The EPED model determines pedestal height and pedestal width by identifying the intersection between the PB constraint and the KBM constraint.

The prescribed inputs of Europed runs are the magnetic equilibrium, the electron density at the pedestal top $n_{e^{\text {ped }}}$, the value of $\beta_{\mathrm{pol}}$, and the temperature and density at the separatrix $T_{e}^{\text {sep }}$ and the relative shift, defined as the distance between position of the pedestal density $n_{e}^{\text {pos }}$ and temperature $T_{e}^{\text {pos }}$.[19] Note that the separatrix density $n_{e}^{\text {sep }}$ is not an input parameter, but is determined by $n_{e}^{\text {pos }}-T_{e}^{\text {pos }}$ and by the density offset applied in the SOL. For the same offset, the increase of the relative shift leads to the increase of $n_{e}^{\text {sep }}$.[20] The value of $n_{e}^{\text {ped }}$ has been set to achieve a volume averaged electron density $\left\langle n_{e}\right\rangle \sim(0.40-0.47) n_{G}$. $T_{i}=T_{e}$ has been assumed in the pedestal. Instead, the value of $\beta_{\text {pol }}$ has been chosen, in an iterative way, in order to match the value predicted by JINTRAC run. In the full power simulations, $n_{e}^{\text {ped }}=1.4 \times 10^{20} \mathrm{~m}^{-3}, \beta_{\mathrm{pol}}=0.60$, a relative shift between $n_{e}^{\text {ped }}$ and $T_{e}^{\text {ped }}$ to obtain $n_{e}^{\text {sep }} \approx 0.25 n_{e}^{\text {ped }}$, and $T_{e}^{\mathrm{sep}}=100 \mathrm{eV}$ values have been set and temperature values at the pedestal top of about $T_{e}^{\text {ped }} \simeq 2.4 \mathrm{keV}$ have been predicted. A more detailed discussion about the Europed input values is addressed in section 3.5.2.

Inside the top of the pedestal, the turbulent heat and particle transport is calculated by Trapped-Gyro-LandauFluid (TGLF) [21, 22, 23, 24], which is a gyrofluid and electromagnetic quasi-linear model with shaped flux surfaces, or by QuaLiKiz (QLK)[25, 26], which is a gyrokinetic and electrostatic quasi-linear transport model with circular flux surfaces. A large amount of work has been made in the last decade to validate these models against experimental results. A wide overview on progress in understanding core transport in tokamaks is presented in [27], including examples of validation of quasi-linear models against present experiments. Some recent TGLF validation carried out for DIII-D and AUG plasma discharges are reported in $[28,29,30,31]$, while recent QLK validation works are presented in $[32,25,33,34,35]$ for hybrid, baseline, and mixed-isotope JET experiments. Bearing in mind that DTT will operate in a $T_{e}>T_{i}$ regime, a particularly relevant validation work is the one presented in [36], with results of both TGLF and QLK modelling of an extensive set of experimental results from AUG and JET-ILW in regimes with high $T_{e} / T_{i}$.

In this paper, the the two most recent versions of TGLF have been used: TGLF SAT1-geo, released in November 2019, featuring an improved description of geometrical effects and calibration against CGYRO non-linear simulations and TGLF SAT2, released in January 2021, featuring further improvements and better agreement with CGYRO as discussed in [37]. In runs with QLK, a recent release of the model [38] with improved TEM treatment thanks to a revised collision operator has been employed. For QLK, in addition to this new official version, an ad hoc version of this model, where the TEM electron heat flux has been multiplied by a factor 2 to match the gyrokinetic simulations described in sect.3.5.4, has also been tested. This ad hoc retuning is physically justified by the fact that the region inside mid-radius in DTT is strongly dominated by TEM, which is challenging for QuaLiKiz. The ad-hoc QuaLiKiz correction is not intended here to be a recommended general prescription, but rather a form of uncertainty quantification by modifying the model to account for known physics deficiencies for this specific case. In all QLK runs, the EM stabilisation mock-up[35] has been turned off because of the $T e \gg T i$ regime of DTT scenarios, which is outside the regime where the mock-up was developed.

For reasons of numerical stability, a small fraction (3\%) of Bohm transport is added to the main turbulent transport. For electron heat transport, which has negligible neoclassical component, an additional diffusivity $\chi=0.5 \mathrm{~m}^{2} / \mathrm{s}$ has been added in the region $\rho_{\text {tor }}<0.2$, where the turbulence level tends to vanish.

In order to set-up the runs in a faster way, simulations with the QLK Neural Network (QLKNN)[39] model have been also carried out, applying the QLKNN-hyper-10D version. This work has been also useful to test the proper functioning of QLKNN in the DTT regime conditions.

The neoclassical transport is calculated by the RomanelliOttaviani model[40] for impurities and NCLASS[41] for main particles.

The toroidal rotation is predicted using a theory-driven empirical model[42, 43], in which the inward momentum pinch is included in the simulation thanks to the construction of a pinch number $R V_{\phi} / \chi_{\phi}$ that has the trend $R V_{\phi} / \chi_{i} \propto-\sqrt{r / R}$ given by [42], is null at the plasma centre, and is $\sim 2.5$ at $\rho_{\text {tor }}=0.4$. The choice of those conditions is based on an analysis of some plasma parameters and the experimental pinch number dependence on those parameters found out in [43]. The Prandtl number $\chi_{\phi} / \chi_{i}$ is fixed at 0.7 , i.e. in the place of a calculated momentum transport coefficient $\chi_{\phi}$ the product $0.7 \chi_{i}$ is used, where $\chi_{i}$ is the ion thermal transport coefficient. The choice of 0.7 accounts empirically also for the component of residual stress due to $E \times B$ shearing, which lowers the nominal Prandtl number. The rotation pedestal has been arbitrarily assumed $10 \mathrm{krad} / \mathrm{s}$ taking from present devices, in any case we note that the simulation is mainly influenced by the rotation gradient, not by its absolute value.

Heating and current drive are modelled self-consistently in JINTRAC runs with suitable codes, as described in 3.4. The particle source from NBI is also calculated, whilst the edge neutral penetration is negligible inside $\rho_{\text {tor }}=0.94$.

In our integrated modelling, Argon (Ar, $A \simeq 40$, $Z=18$ ) and Tungsten (W, $A \simeq 184, Z=74$ ) are included as impurities. Argon is a seeding gas used to enlarge the edge radiative dissipation decreasing the divertor power load, while Tungsten comes from the divertor. 
In JINTRAC runs, impurity densities and radiation are simulated up to the separatrix with SANCO[44]. For both gases, all ionisation states are treated separately by SANCO. In order to conserve the particle number equal to the initial value, escape velocity, neutral influx and recycling factor are set null. A radially constant effective charge equal to $Z_{\text {eff }}=\sum_{i} Z_{i}^{2} n_{i} / n_{e}=1.7$ (sum over ion species) and a density ratio $n_{W} / n_{A r}=0.05$ are used as initial conditions.

Evaluating the neutron rate is a key point in the tokamak design, because the neutron shields have to be able to withstand the neutron loads. In the JINTRAC simulations, the total neutron number is calculated as sum of neutrons produced by the fusion reactions between two thermal nuclei, between a thermal nucleus and a fast nucleus of the NBI beam, and between a thermal nucleus and a fast nucleus of the ICRH minority species.

Sawteeth and ELMs are not included in the modelling, with the exception of the simulations described in sect. 3.5.3 where a continuous model for ELMs has been used. The absence of sawteeth implies that the profiles presented here would correspond to the saturated recovery after a sawtooth crash.

\section{$3.2 \quad$ JINTRAC \& ASTRA}

The DTT simulations have mainly been carried out with the JINTRAC suite with the JETTO[45] transport solver. The JINTRAC system includes several interfaced tokamak-physics codes ( $\sim 25$ modules) and has been used extensively for decades on experimental data of different tokamaks and to predict future devices. The $1.5 \mathrm{D}$ core plasma fluid code JETTO is the central part of JINTRAC, designed to calculate plasma profiles up to the separatrix. The JINTRAC suite has been used for full physics simulations of DTT using QLK or QLKNN, predicting current density and equilibrium, temperatures, densities (main ion and impurities), rotation, and heating, as described in sect. 3.1.

In addition, the ASTRA transport solver has also been used, within an iterative ASTRA-JINTRAC scheme devised for some high complexity cases with TGLF as turbulent transport model, due to the low speed of JINTRAC TGLF runs with DTT parameters. The starting point of this mixed method is running a JETTO simulation with QLK and use the resulting profiles as inputs for ASTRA. Then, an ASTRA run predicts temperatures and density with fixed current density, heating, toroidal rotation, impurities, and radiative power, taken from JINTRAC. In this run, the equilibrium is solved self-consistently by the SPIDER[46] code. Impurities are included but not evolved in ASTRA; their profiles are set proportional to the electron density $n_{e}$ with a constant that reflects the JINTRAC settings. The impurity ionisation profiles are the ones provided by JINTRAC. As third step, the ASTRA profiles of density and temperatures are kept fixed

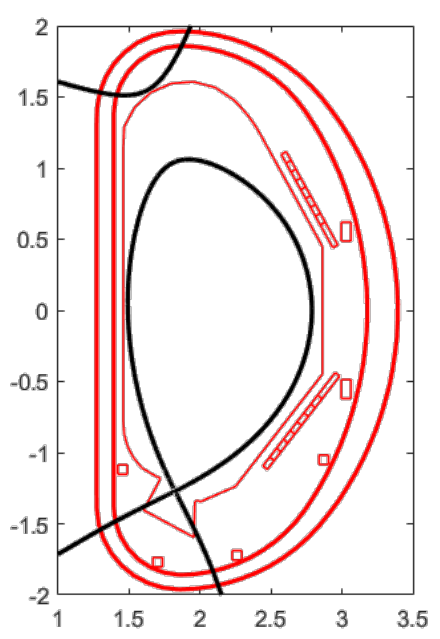

Figure 2: Plasma shape of the SN DTT scenario.

in a new JETTO run aimed at recalculating heating and safety factor profiles. The second and the third phases are repeated until convergence. This mixed ASTRAJINTRAC approach is quite efficient, because ASTRA TGLF simulations are much faster than JETTO TGLF runs and one iteration usually is enough.

\subsection{Equilibrium}

The expected standard operational points of DTT (with $R_{0}=2.14 \mathrm{~m}$ ) in terms of on-axis toroidal magnetic field $B_{t}$ and plasma current $I_{p l}$ are the following:

- full current and full field operational point, with $I_{p l}=5.5 \mathrm{MA}$ and $B_{t}=6 \mathrm{~T}$;

- reduced current and full field operational point, with $I_{p l}=4.0 \mathrm{MA}$ and $B_{t}=6 \mathrm{~T}$;

- reduced current and half field operational point, with $I_{p l}=2.0 \mathrm{MA}$ and $B_{t}=3 \mathrm{~T}$.

For these simulations, reference DTT plasma equilibria with average triangularity $\langle\delta\rangle \simeq 0.3$, major radius $R_{0}=$ $2.14 \mathrm{~m}$, and minor radius $a=0.65 \mathrm{~m}$ for each of these standard operational points have been provided by the free boundary CREATE-NL[47] solver.

In the JETTO simulations, the MHD equilibrium is self-consistently recalculated 3 times per second by the equilibrium solver ESCO integrated in the suite. The plasma boundary is kept fixed to the CREATE-NL reference one. The plasma shape of the SN DTT scenario at the full current and full field operational point is shown in figure 2.

At the flux surface that contains the $95 \%$ of the poloidal flux, performed simulations of DTT full power scenarios returned triangularity values in the range $\delta_{95}=0.29-0.31$, 
elongation values in the range $k_{95}=1.66-1.69$, and safety factor values in the range $q_{95}=2.6-2.7$.

\subsection{Heating and Current Drive (HCD)}

Since DTT will be equipped with three auxiliary heating systems, the integrated simulations of this device feature high complexity level. The ECRH, ICRH, and NBI power depositions are computed several times during the runs, including the synergy effects.

In the heating configuration of full power option D, which has become the new reference option for the full power scenario, there are $4 \mathrm{ECRH}$ clusters. Each cluster is composed of 8 gyrotrons at $170 \mathrm{GHz}$, with an installed power of 1-1.2 MW from each gyrotron. Depending on the access port, these 8 gyrotrons are divided into 2 upper (UP) gyrotrons, 3 equatorial top (EQT) gyrotrons, and 3 equatorial bottom (EQB) gyrotrons. A loss factor before launchers of 0.9 is evaluated, leading the ECRH power at plasma to around $30.2 \mathrm{MW}$.

The ICRH system of DTT is designed to operate in the frequency range $60-90 \mathrm{MHz}$. In the reference $B_{t}=6 \mathrm{~T}$ scenario, the cyclotron resonances of ${ }^{3} \mathrm{He}$ and $\mathrm{H}$ minorities are located on-axis when the ICRH frequency is $60 \mathrm{MHz}$ or $90 \mathrm{MHz}$ respectively. The system is devised in modular units, placed in equatorial ports, and each module is based on a pair of 2-strap antennas. In order to better cope with abrupt coupling changes because of $\mathrm{L}-\mathrm{H}$ transitions or ELMs, the 2 antennas of a module are fed in parallel. Since the power supplied by each RF antenna is $2 \mathrm{MW}$, supposing an efficiency of 0.75 (typical efficiencies for transmission lines and antenna coupling are $80 \%$ and $90 \%$ ), the ICRH coupled power is $\sim 1.5 \mathrm{MW}$ per antenna. The installation of the first RF module is scheduled for the initial phase. Depending on the full power option choice, an upgrade with one or two modules more may be realised. Particularly, the full power option D heating configuration includes two ICRH modules.

Due to the high DTT densities, to allow a central NBI power deposition during the flat-top plasma discharge phase, a negative ion-based NBI system at high energies $E>300 \mathrm{keV}$ must be used in DTT. During early current ramp-up, late current ramp-down, and low current scenarios the employment of the NBI system must be cautiously evaluated, in order to avoid shine-through risks. Moreover, an important feature of the NBI system is its current drive capability and central fuelling. The full power option D heating configuration includes only $1 \mathrm{NBI}$ injector, which provides $\sim 10.0 \mathrm{MW}$ of power to the plasma, with a $500 \mathrm{keV}$ Deuterium beam. In the day-1 scenario, this injector will be used at reduced energy $(\sim 400 \mathrm{keV})$ supplying a NBI power amount $\leq 7.5 \mathrm{MW}$ to the plasma.

The RF antenna, NBI injectors, and ECRH gyrotrons have been configured within the JINTRAC suite.

The ECRH power deposition is calculated every $0.25 \mathrm{~s}$ by the GRAY code[48]. Since DTT gyrotrons are too numerous to be included separately in GRAY (the maximum number is 20), they have been grouped in subsets. In the full power option D run, 12 beams are used (2 UP beams, 5 EQT beams, and 5 EQB beams). Each beam is simulated by the sum of one central ray and 160 rays arranged on 10 concentric rings and has a toroidal angle equal to $2^{\circ}$. In simulations of full power and day-1 scenarios all beams are injected in O-mode. Due to the lower magnetic field value, in the day-0 scenario the EC power is expected to be absorbed at second harmonic. Since the O-mode polarisation is known to be less efficient at second harmonic, the ECRH system will be used in X-mode for the day- 0 case to maximise the absorption. The poloidal angles have been set in the following ranges: $43^{\circ}-44^{\circ}$ for UP beams, $2^{\circ}-6^{\circ}$ for EQT beams, and $\left(-13^{\circ}\right)-\left(-15^{\circ}\right)$ for EQB beams.

The NBI power deposition is calculated by the PENCIL code[49]. Since the DTT NBI source is composed by negative ions, all beam particles are injected at the nominal energy. Hence, in PENCIL the full energy fraction has been set equal to 1 . The total loss of NBI fast particles, considering both prompt and ripple losses, has been assessed at $\sim 4 \%$ in [50] and hence has resulted negligible.

The PION[51, 52, 53] code calculates the ICRH power deposition, including the synergy effects with NBI. In the performed simulations Hydrogen has always been used as minority species with a concentration of $5 \%$ and the $\mathrm{RF}$ frequency has been set to $90 \mathrm{MHz}$. So, the cyclotron resonance is located where the magnetic field is equal to $B \simeq 5.9$ T, i.e. at $\rho_{\text {tor }} \sim 0.15$.

\subsection{Full Power Option D scenario}

The so-called option $\mathrm{D}$ has been selected as the new reference configuration for the Full Power (FP) scenario. In this configuration, auxiliary heating systems deliver a total power of $\sim 46 \mathrm{MW}$ to the plasma: $\sim 10.0 \mathrm{MW}$ from the NBI system, $\sim 6.0 \mathrm{MW}$ from the ICRH system, and $\sim 30.2 \mathrm{MW}$ from the ECRH system.

\subsubsection{Simulations with QLK or TGLF of full power option $\mathrm{D}$ scenario}

The integrated modelling of a steady-state Deuterium plasma in the FP option D scenario has been performed using both the standard QLK model and an ad hoc QLK version in a JETTO run and the TGLF SAT1-geo or SAT2 model with the JINTRAC-ASTRA approach.

The electron temperature $T_{e}$, ion temperature $T_{i}$, electron density $n_{e}$, toroidal rotation $\omega_{\text {tor }}$, and safety factor $q$ radial profiles obtained by these four runs are shown in figure 3. The radial profiles of all power densities and those of the total electron and ion powers are displayed in figure 4 (a) and 4(b) respectively, only for the standard 


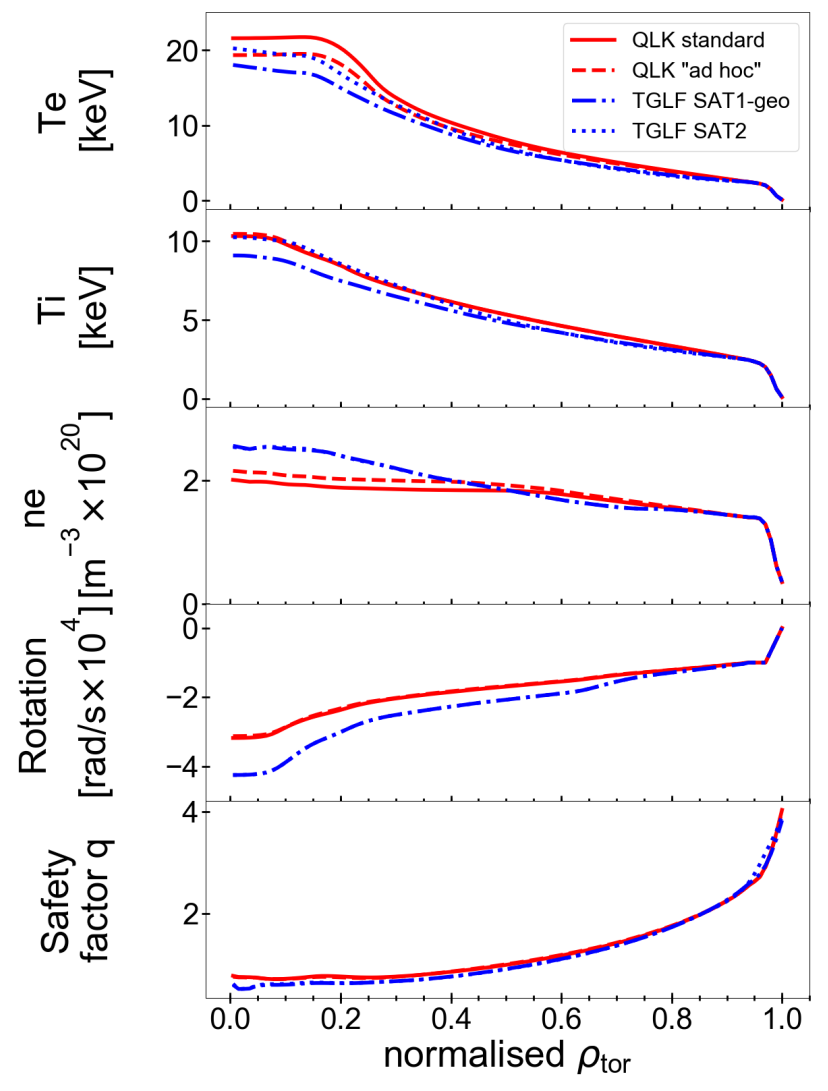

Figure 3: Steady-state radial profiles of the electron and ion temperatures, electron density, toroidal rotation, and safety factor of the FP option D scenario, with turbulent transport calculated by TGLF SAT1-geo (blue dash-dot line) or SAT2 (blue dotted line) or by standard QLK (red solid line) or ad hoc QLK with TEM electron heat flux multiplied by a factor 2 (red dashed line).
QLK simulation. The power density and total power profiles resulting from the other three runs are similar in shape and size. The current density radial profile and its main contribution are shown in figure 5 .

From figure 3 , we notice that the profiles of $T_{e}$ and $T_{i}$ reach maximum values in the range of $18.0-21.6 \mathrm{keV}$ and in the range of $9.1-10.4 \mathrm{keV}$ respectively. There is a good agreement between TGLF and QLK temperature profiles, which are a bit smaller in the TGLF SAT1-geo run, up to differences of the order of $\sim 15-20 \%$ at the plasma centre, because of a slight difference in the temperature gradient in the region $0.65 \lesssim \rho_{\text {tor }} \leq 0.94$. Electron densities of the two models present a good agreement for $\rho_{\text {tor }} \gtrsim 0.5$, but a non-negligible discrepancy appears inside. Particularly, $n_{e}$ has a moderately peaked profile in the TGLF simulation, while the QLK density profile is extremely flat in the inner half of the plasma. Due to a quite different $n_{e}$ gradient in $0.2 \lesssim \rho_{\text {tor }} \lesssim 0.5$, the maximum $n_{e}$ value sweeps from $2.0 \times 10^{20} \mathrm{~m}^{3}$ to $2.6 \times 10^{20} \mathrm{~m}^{3}$.

In order to identify the most reliable prediction and to explain the difference of density peaking between TGLF and QLK, a benchmark work of the two quasi-linear models against the gyrokinetic model GENE[54,55] in the DTT parameter range has been performed and its results are displayed in sect. 3.5.4.

Comparing the modelling results between the standard QLK version and the QLK version with $2 \times q_{e \text {,TEM }}$, we noticed that the "ad hoc" model introduced small variations in the right direction. Particularly, the density peaking is a bit increased (closer to the TGLF one) in the "ad hoc" QLK density profiles. Nevertheless, these improvements are too small to justify the employment of this QLK version in further simulations.

With both QLK and TGLF models, it turns out that DTT is characterised by $T_{e}$ significantly larger than $T_{i}$, particularly in the inner half of the plasma. This is due to the very large and localised ECH power density $\left(P_{\mathrm{ECH} e} \sim 1.1 \times 10^{7} \mathrm{~W} / \mathrm{m}^{3}\right)$, and to the fact that $T_{e} / T_{i}$ is a key factor determining the ion critical gradient $\left(R / L_{T}\right)_{\text {crit }}[56,57]$, lowering it for increasing $T_{e} / T_{i}$. The low ITG threshold in presence of a high ion stiffness then prevents $T_{i}$ from peaking. This behaviour is in line with several observations in nowadays tokamaks or stellarators with high electron heating, see for example the recent work in [58]. Instead $T_{e}$ is largely determined by TEMs, which exhibit much lower stiffness and typically higher $\left(R / L_{T_{e}}\right)$ thresholds, so that a higher $T_{e}$ peaking can be reached. The ITG modes and TEMs are dominant in these plasmas, while ETG modes[59], which are included in the integrated simulations, do not play an important role, due to the high $T_{e} / T_{i}$. The ETG unimportance resulted evident from both stand-alone runs and profile simulations performed with TGLF and with QLK with/without ETG inclusion. Moreover, the linear gyrokinetic runs carried out with GENE also confirmed the lack of the ETG contribution. 


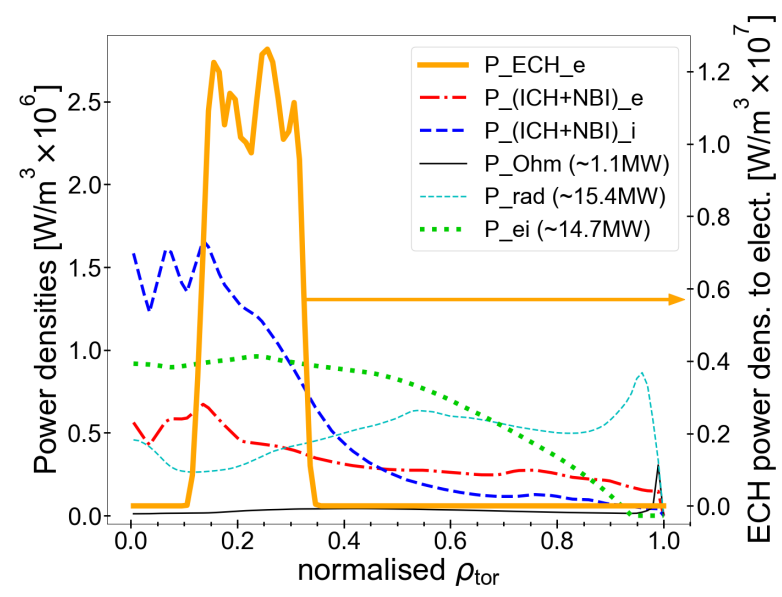

(a) Power densities.

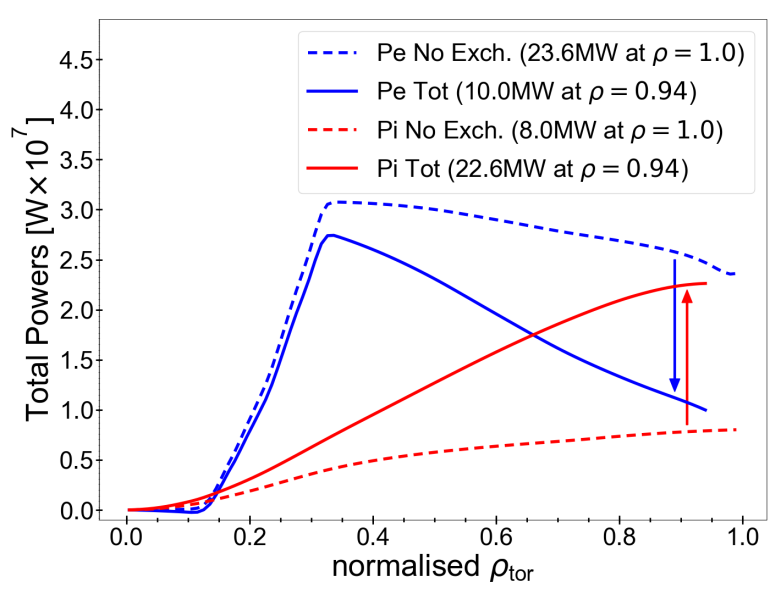

(b) Volume integrated powers.

Figure 4: (a) Radial profiles of power densities: ECRH power deposited to electrons $P_{\mathrm{ECH}} e$, NBI and ICRH power deposited to electrons $P_{(\mathrm{ICH}+\mathrm{NBI})}$, NBI and ICRH power deposited to ions $P_{(\mathrm{ICH}+\mathrm{NBI})}$, Ohmic power $P_{\mathrm{Ohm}}$, radiative power $P_{\text {rad }}$, and thermal exchange power between electrons and ions $P_{e i}$. (b) Radial profiles of electron and ion total powers including or not including the thermal exchange power between species.

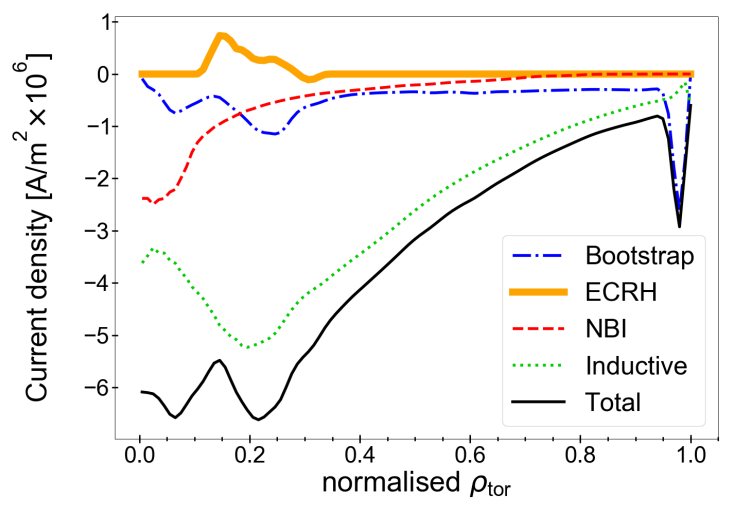

Figure 5: Radial profiles of the total current density and of its main contributions.

Locally, in the DTT FP scenario, electron heating dominates in the inner plasma region, as evidenced by figure 4(b). Since the ion channel is very stiff and bound to a low critical gradient due to $T_{e} / T_{i}>1$, ions represent a big power sink through collisional exchange. The ion temperature profile results stuck near the threshold irrespective of the large amount of supplied power.

Globally, the core radiated power $P_{\text {rad }} \approx 15.4-17.8 \mathrm{MW}$ is about the $35 \%$ of the total power, the Ohmic power $P_{\mathrm{Ohm}} \approx 1.1 \mathrm{MW}$ is quite negligible, and a large amount of power $\left(13.7 \mathrm{MW} \lesssim P_{e i} \lesssim 14.7 \mathrm{MW}\right)$ is exchanged from electrons to ions because of the collisional coupling. Therefore, although the external electron power $P_{\text {ext }} \approx 38 \mathrm{MW}$ is much bigger than the external ion power $P_{i \text { ext }} \approx 8 \mathrm{MW}$, globally the total electron power $P_{\text {etot }} \approx 9.5-10.4 \mathrm{MW}$ is much lower than the total ion one $P_{i \text { tot }} \approx 21.8-22.6 \mathrm{MW}$. In DTT, the collisional time is higher than the confinement time, so the collisional exchange is not enough to equilibrate $T_{e}$ and $T_{i}$. As things stand, obtaining an ion temperature profile as high as possible would be beneficial; to achieve this, one would have to find ways of reducing the ion stiffness or increasing the ITG threshold, besides having more central ion power. The choice of the option D as the FP reference scenario has been based on this principle. Particularly, some electromagnetic (EM) stabilisation effects are known to reduce ion stiffness and they can be increased by the presence of fast ions. $[60,61]$ These considerations have led to the choice of increasing the ICRH power to the maximum technically feasible and increase the NBI energy with respect to the original proposal described in [6].

From figure 3, we also note that the safety factor value at the flux surface that contains the $95 \%$ of the poloidal flux is quite low $q_{95} \approx 2.5-2.6$. An increased disruptivity is observed in plasma with such low $q_{95}$, e.g. [62]. As a consequence, the DTT team decided to enlarge the DTT major radius from $R_{0}=2.14 \mathrm{~m}$ to $R_{0}=2.19 \mathrm{~m}$ and its minor radius from $a=0.65 \mathrm{~m}$ to $a=0.70 \mathrm{~m}$, to bring $q_{95}$ nearer to 3 .

Within the JINTRAC runs, the contribution of impurities is calculated by SANCO, using neoclassical transport and turbulent transport from QLK. In figure 6, the density profiles of impurities and the profile of the effective charge $Z_{\text {eff }}$ are displayed for the QLK case with solid lines. The TGLF simulations in ASTRA do not evolve impurity species, but only take into account their effects assuming 


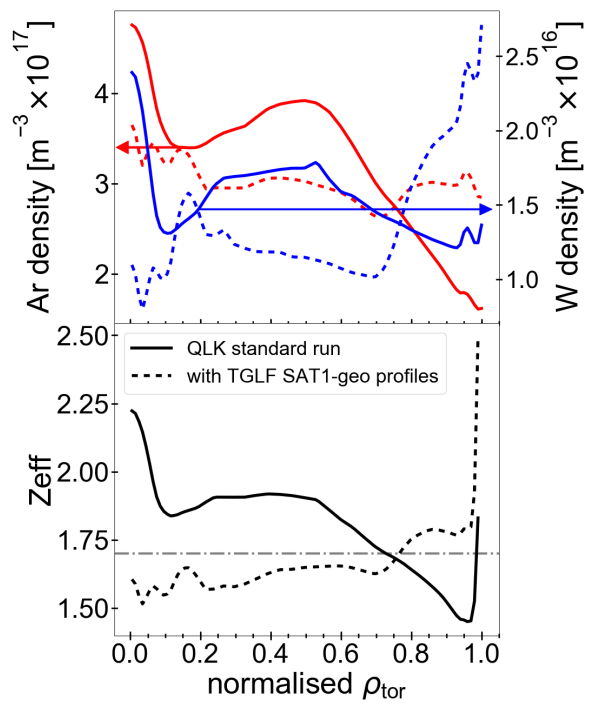

Figure 6: Radial profiles of the impurity densities (Argon in red, Tungsten in blue) and of effective charge $Z_{\text {eff. }}$. The standard QLK case results (solid lines) are compared to an assessment of the impurity and $Z_{\text {eff }}$ profiles in presence of TGLF SAT1-geo predicted profiles carried out with JETTO/SANCO and QLK (dashed lines).

the $n_{e}$ profile shape. Nevertheless, it is interesting to get an idea of which argon and tungsten densities would be computed by QLK for $T_{e}, T_{i}$, and $n_{e}$ values such as those of the TGLF case. Thus, a JETTO run with interpretative $T_{e}, T_{i}$, and $n_{D}$ profiles equal to the TGLF SAT1-geo case has been performed, including SANCO calculations for impurity densities and using QLK as turbulent transport model for the impurities. This allows us to estimate the effect of the TGLF electron density peaking on the impurity profiles, as shown in figure 6 with dashed lines. Argon and Tungsten densities amount to $n_{\mathrm{Ar}} / n_{e} \approx 0.28 \%$ and $n_{\mathrm{W}} / n_{e} \approx 0.014 \%$ respectively. We observe some penetration of the impurities into the core with both models. In future works, other possible seeding gasses will be tested in place of Argon to investigate their effect on the edge radiative dissipation.

The largest neutron loads to be coped with obviously occur in the FP scenario. The more challenging prospect in this respect is represented by the TGLF SAT2 FP run, where the maximum value of neutron rate is reached. The total neutron density rate and the radial profiles of its three contributions shown in figure 7 refer to the run outcome with TGLF SAT2 profiles. Integrating over all the profile up to the separatrix, the total neutron rate in this case amounts to $1.47 \times 10^{17}$ neutrons/s, resulting compatible with the present design of neutron shields with a good safety margin. The neutron density rates obtained from simulations with standard or ad hoc QLK or with TGLF SAT1-geo profiles are a bit lower, but definitely

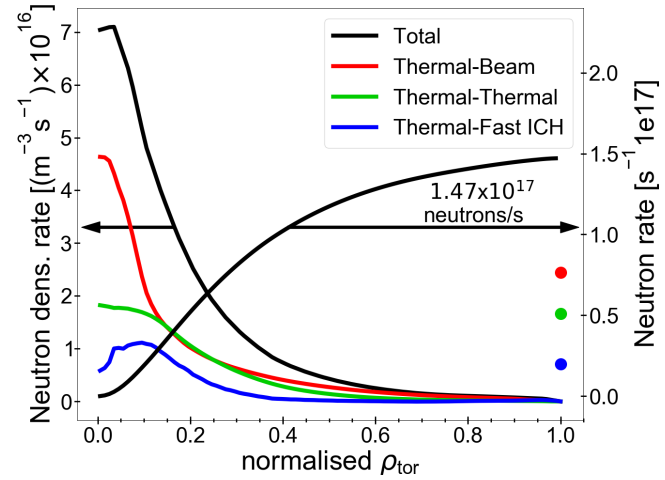

Figure 7: Radial profiles of neutron density rates, where neutrons are produced by fusion reactions between: two thermal nuclei (green), between a thermal nucleus and a fast nucleus of the NBI beam (red), and between a thermal nucleus and a fast nucleus of the ICRH minority species (blue), any pair of nuclei (black). The radial profile of the total neutron rate is also displayed (black), with points indicating the three contributions to it. These profiles refer to the TGLF SAT2 FP case.

similar in shape, to give total neutron rates in the range of $1.29-1.36 \times 10^{17}$ neutrons/s. In all cases, the largest contribution is given by the fusion reactions between NBI fast Deuterium and thermal Deuterium, but the thermalthermal neutrons are also very significant.

In figure 8 the density and energy density radial profiles of energetic particles are shown for the standard QLK run. The EP profiles in the TGLF SAT2, TGLF SAT1geo, and ad hoc QLK cases present similar shapes. In the FP reference scenario, the energy fraction owned by the EPs amounts to $W_{\mathrm{EP}} / W_{\text {tot }} \approx 6.5-7.7 \%$.

For the sake of completeness, the table 2 presents the main dimensionless physical quantities of this scenario. Particularly, the total radiation from the plasma inside

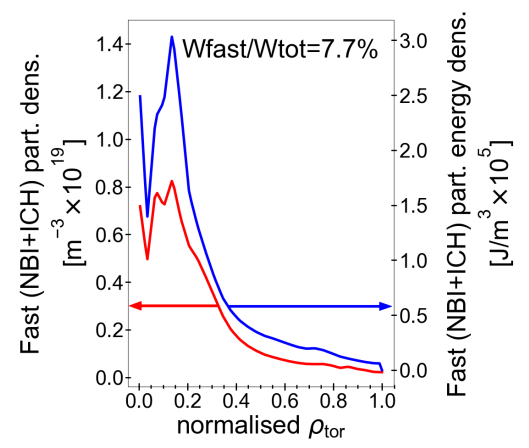

Figure 8: Radial profiles of density and energy density of energetic particles due to the both NBI and ICRH systems. 
Table 2: FP option D scenario dimensionless quantities.

\begin{tabular}{lcccc}
\hline & $\begin{array}{c}\text { TGLF } \\
\text { SAT1-geo }\end{array}$ & $\begin{array}{r}\text { TGLF } \\
\text { SAT2 }\end{array}$ & $\begin{array}{c}\text { QLK } \\
\text { standard }\end{array}$ & $\begin{array}{c}\text { QLK } \\
\text { ad hoc }\end{array}$ \\
\hline$\tau_{E}\left(P=P_{\text {tot }}\right)$ & 0.25 & 0.27 & 0.27 & 0.28 \\
\hline$\tau_{E}\left(P=P_{\text {sep }}\right)$ & 0.43 & 0.42 & 0.41 & 0.43 \\
\hline$H_{98}\left(P=P_{\text {sep }}\right)$ & 0.95 & 1.00 & 0.99 & 0.98 \\
\hline$\beta_{\mathrm{N}_{\text {therm }}}[\%]$ & 1.39 & 1.47 & 1.49 & 1.51 \\
\hline$\beta_{\mathrm{N}_{\text {tot }}}[\%]$ & 1.49 & 1.57 & 1.62 & 1.62 \\
\hline$W_{\mathrm{EP}} / W_{\text {tot }}[\%]$ & 6.5 & 6.7 & 7.7 & 6.7 \\
\hline$\left\langle n_{e}\right\rangle / n_{G}$ & 0.44 & 0.44 & 0.40 & 0.42 \\
\hline
\end{tabular}

the separatrix can be subtracted or not from the input power when calculating the confinement, yielding the two $\tau_{E}$ values in table.

\subsubsection{Pedestal variations}

The core-edge integration is a key point in the DTT integrated modelling work. Since the pedestal points are used as boundary conditions in the JETTO/ASTRA simulations, for the FP scenario we investigated the pedestal height variability depending on the Europed run inputs to examine robustness of the major results of our analysis. Some Europed inputs (such as $I_{p l}, B_{t}, R_{0}, \ldots$ ) are imposed by the selected scenario and by general DTT parameters, therefore we have not performed sensitivity tests on these. We have tested the sensitivity to $\beta$ vaues in the range $0.4 \lesssim \beta_{\text {pol }} \lesssim 0.8$ and verified that the effect is small. Then, the $\beta_{\text {pol }}$ value has been selected iteratively to match the JINTRAC predicted value.

It has also been checked that $T^{\mathrm{sep}}$ value variations in the range of $100-200 \mathrm{eV}$ did not lead to significant changes in the pedestal values. Substituting Argon with an other impurity (for instance with Neon as seeding gas) or varying the $Z_{\text {eff }}$ value around the reference value do not impact effectively the pedestal height. All these small $T_{e}^{\text {ped }}$ variations fall back into a typical accuracy of around $\pm 20 \%$ of the EPED model when the pedestal is peeling-ballooning limited.[63]

Although modelling the direct effect of the gas on the pedestal is outside the scope of this work, the effect of the gas is expected to increase $n_{e, \text { sep }}$ and shift the density position $n_{e, \text { pos }}$ outwards, as observed in AUG, JET-ILW, TCV, and DIII-D [64, 19, 65, 66, 67]. Therefore, we have assessed the gas effect on the pedestal by testing the impact of the density position on the predicted $T_{e^{\text {ped }}}$.

A null relative shift $\left(n_{e, \text { pos }}=T_{e, \text { pos }}\right)$, is the standard assumption in the basic EPED model and has been used throughout the paper. Assuming that the density has the same position as the temperature (corresponding to $n_{e, \text { sep }} / n_{e, \text { ped }} \approx 0.25$, i.e. to $\left.n_{e, \text { sep }} \approx 3.5 \times 10^{19} / \mathrm{m}^{3}\right)$, the
Europed run predicts $T_{e, \text { ped }} \approx 2.2 \mathrm{keV}$.

However, relatively recent results shows that tipically $n_{e}^{\text {pos }}>T_{e}^{\text {pos }}$, with values of the shift higher than $\approx$ $0.002 \psi_{N} \cdot[19,64]$ The possible effect of $n_{e}^{\text {pos }}>T_{e}^{\text {pos }}$ has been tested, as displayed in figure 9 .

By increasing the relative shift, $T_{e^{\text {ped }}}$ decreases till a saturation is reached above the relative shift $\approx 0.01 \psi_{N}$ (corresponding to $n_{e, \text { sep }} / n_{e, \text { ped }} \approx 0.5$, i.e. to $n_{e, \text { sep }} \approx$ $\left.7 \times 10^{19} / \mathrm{m}^{3}\right)$. The saturation is related to the effect of the density position on the pressure position, as discussed in [20]. In DTT $n_{\mathrm{e}, \mathrm{sep}} / n_{\mathrm{e}, \mathrm{ped}} \lesssim 0.5$ is expected, and in the main simulations of this work $n_{\mathrm{e}, \mathrm{sep}} / n_{\mathrm{e}, \mathrm{ped}} \approx$ $0.2-0.3$. With this value, the EPED model is rather accurate (within $20 \%$ ). The most recent results obtained in JET show that the shortfall compared to EPED occurs at relatively high separatrix density (approximately $n_{\mathrm{e}, \mathrm{sep}} / n_{\mathrm{e} \text {,ped }}>0.5$, with significant discrepancies that occur above 0.6).[68] Nonetheless, to test the effect of possible higher $n_{\mathrm{e}, \mathrm{sep}} / n_{\mathrm{e} \text {,ped }}$ an Europed simulation has been done also at $n_{\mathrm{e}, \mathrm{sep}} / n_{\mathrm{e} \text {,ped }} \approx 0.5-0.6$. This value is expected to be already relative high for DTT, however not high enough to lead to major problems with the reliability of the EPED predictions.[68] Above $n_{\mathrm{e}, \mathrm{sep}} / n_{\mathrm{e}, \mathrm{ped}} \approx$ $0.5-0.6$, there is presently no model to assess reliably the effect of the gas rate on the pedestal.

The minimum temperature reached with the highest relative shift of $0.0125 \psi_{N}$ (with $n_{e, \text { sep }} \approx 8 \times 10^{19} / \mathrm{m}^{3}$, i.e. with $\left.n_{e, \text { sep }} / n_{e, \text { ped }} \approx 0.6\right)$ is $T_{e \text {,ped }} \approx 1.7 \mathrm{keV}$, so approximately $500 \mathrm{eV}$ lower than the reference case.

In order to test the effects of the pedestal variations due to different relative shift values on plasma profiles, we repeated the standard QLK run of the FP reference scenario (with null relative shift) but setting the pedestal points calculated by EPED with a relative shift of $0.0125 \psi_{N}$. Temperature and density radial profiles of these two simulations are compared in figure 10. We observe that the temperature value reduction at the top of the pedestal propagates inwards up to the plasma centre, but it is more interesting to notice the increased density peaking as a consequence of a non-null pedestal relative shift.

Overall however the scenario predictions are not significantly affected.

\subsubsection{Fuelling issues}

In order to evaluate whether the predicted density profiles can be sustained by only gas puff or a pellet fuelling system is required, the level of edge neutrals required to operate in the FP option D scenario without pellets has been investigated.

The standard QLK run of sect. 3.5.1 has been extended up to the separatrix by replacing the fixed pedestal externally calculated by Europed with results of a suitable Edge Transport Barrier (ETB) tuned to reproduce the Europed pedestal.

To calculate the neutral source, the FRANTIC[69] code 

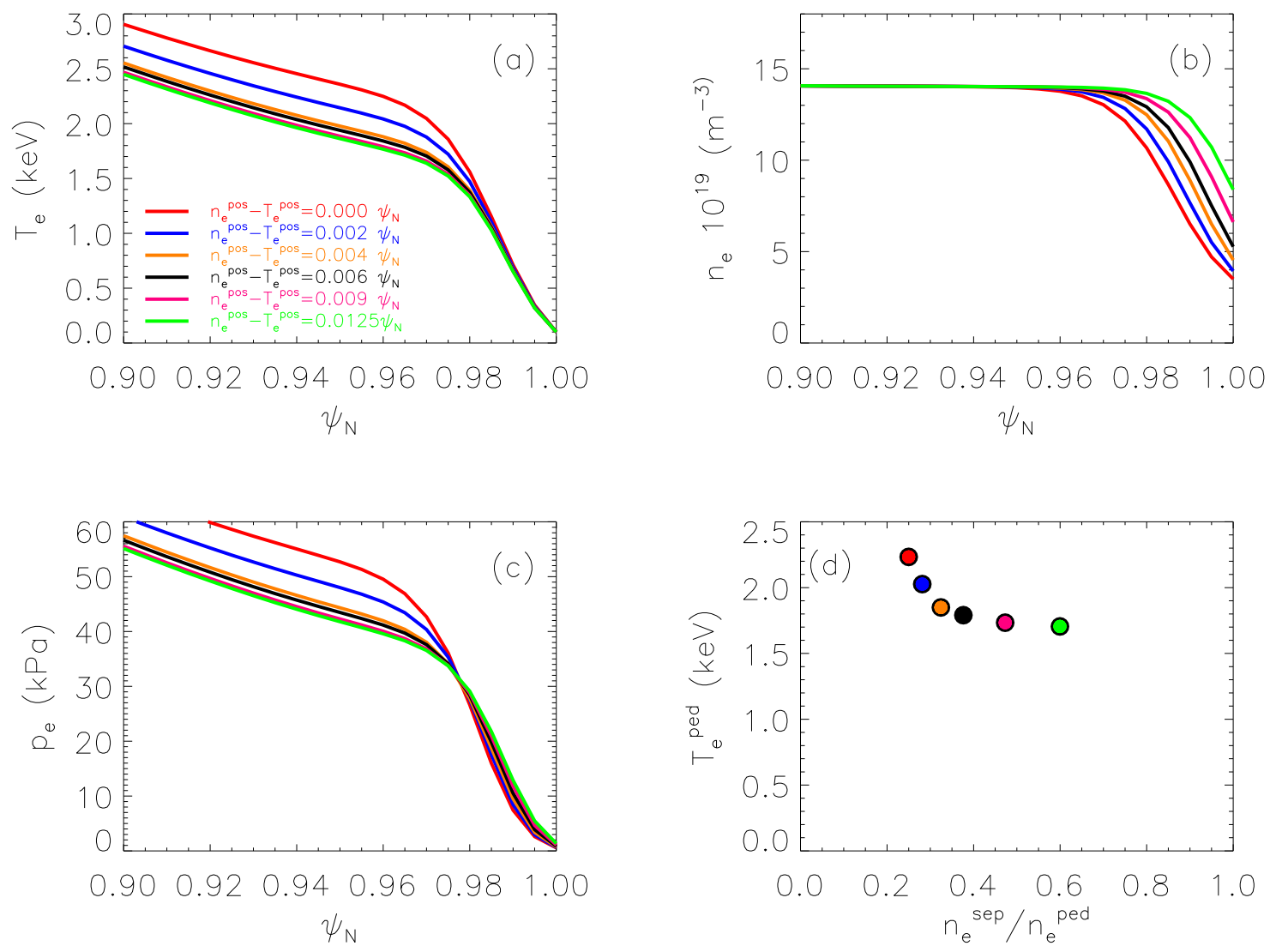

Figure 9: Profiles of (a) electron temperature, (b) density, and (c) pressure in the pedestal region for different values of relative shift $\left(n_{\text {pos }}-T_{\text {pos }}\right)\left(\psi_{N}\right)$. (d) Electron temperature at the pedestal top $T_{e^{\text {ped }}}$ as a function of $n_{e, \text { sep }} / n_{e, \text { ped }}$.

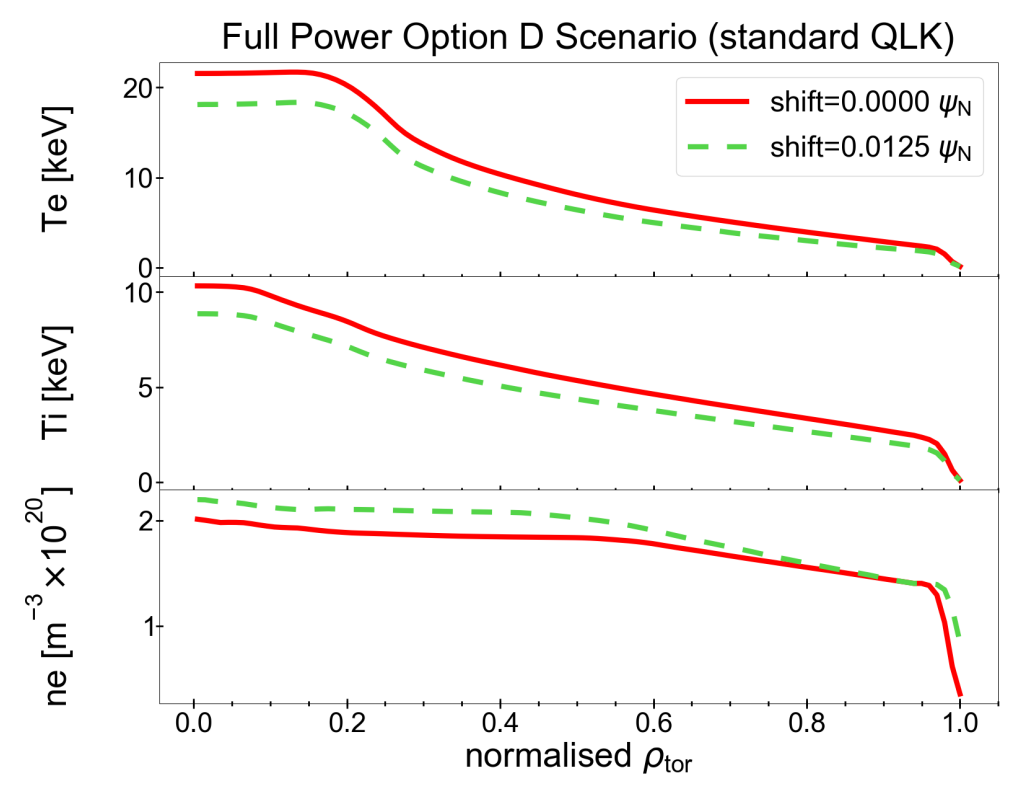

Figure 10: Radial profiles of electron temperature, ion temperature, and electron density predicted by JETTO runs using the standard QLK model for different values of relative shift (the red lines correspond to a null shift, while the green lines correspond to a shift of $0.0125 \psi_{N}$ ). 


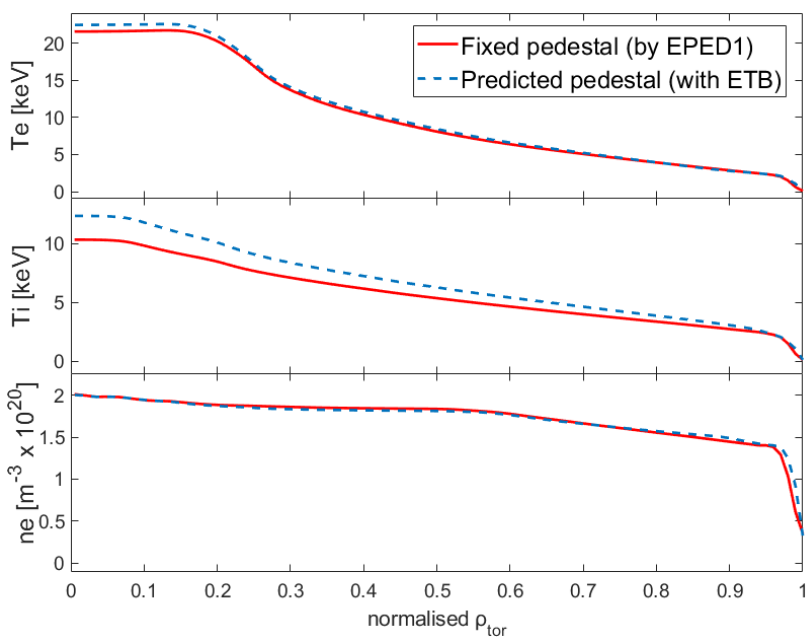

(a) Whole radial profiles.

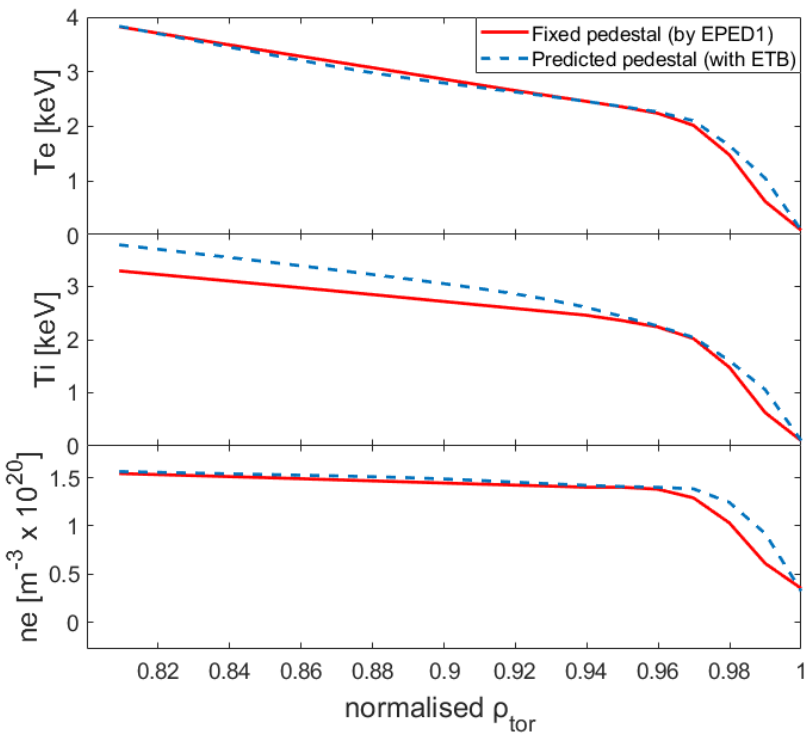

(b) Profile zoom in the pedestal region.

Figure 11: Comparison of $T_{e}, T_{i}$, and $n_{e}$ radial profiles between the standard QLK case with fixed pedestal of sect. 3.5.1 (solid red lines) and the new standard QLK case with the moulded ETB (dashed blue lines).

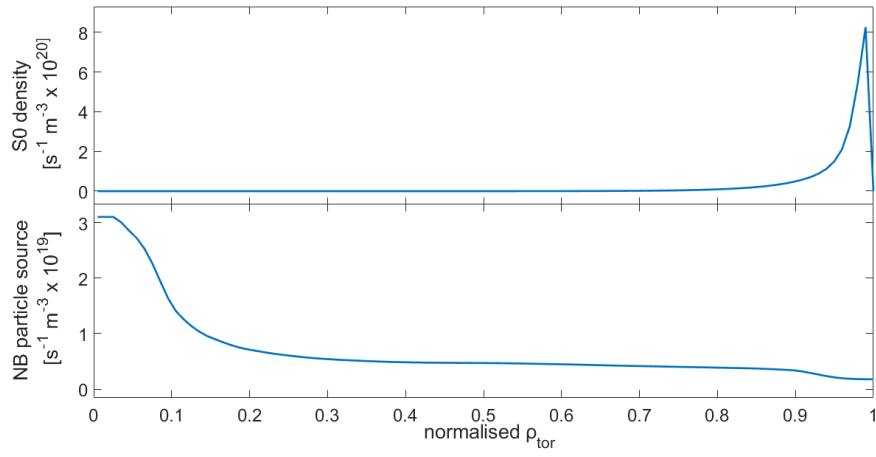

Figure 12: Radial profile of the source of neutrals from the edge and radial profile of the NBI particle source.

has been included in the simulation, setting a feedback control of gas puffing to reach the electron density value expected at the TOB (Top Of Barrier) with null recycling. The ETB transport coefficients are arranged to obtain the temperature at the top of the pedestal as close as possible to the Europed values, thanks to a continuous ELM model. In figure 11, the $T_{e}, T_{i}$, and $n_{e}$ radial profiles resulting from this adjustment work are compared to the profiles of the QLK case with fixed pedestal to show the good agreement between them.

The neutral penetration into the plasma evaluated by FRANTIC is adequate for fuelling, since the neutral density rate is up to $\rho_{\text {tor }} \sim 0.8$, as displayed in figure 12 . The NBI contribution to the neutral source is small.

To reach the density value at the TOB which allows to have $\left\langle n_{e}\right\rangle \sim 0.4 n_{G}$ in the FP reference scenario, a neutral flux level of about $0.36 \times 10^{22}$ particles/s at the separatrix is required.

The dependence of neutral penetration across the separatrix as a function of deuterium fuelling was found starting from results obtained in [70] with the edge code SOLEDGE2D-EIRENE[71, 72]. A scan on fuelling was performed starting from a detached case. Results are shown in figure 13; particularly, $\sim 5 \times 10^{22}$ particles/s is the deuterium fuelling corresponding to the required neutral flux at the separatrix. This entails that we should need a gas puffing and pumping system capable of supplying and pumping at least $\sim 5 \times 10^{22}$ particles/s, which is near to the feasibility limit.

Due to this marginality, and in order to avoid degrading the edge plasma with extremely high pas puff rates, a pellet injection system is deemed useful as a fuelling method in DTT to minimise the operational risk.

A modelling work of pellets is just started. In addition, a work of core-pedestal-SOL integrated modelling, totally self-consitent in terms of temperature and density profiles, fluxes, and transport coefficients, is envisaged as future development. 


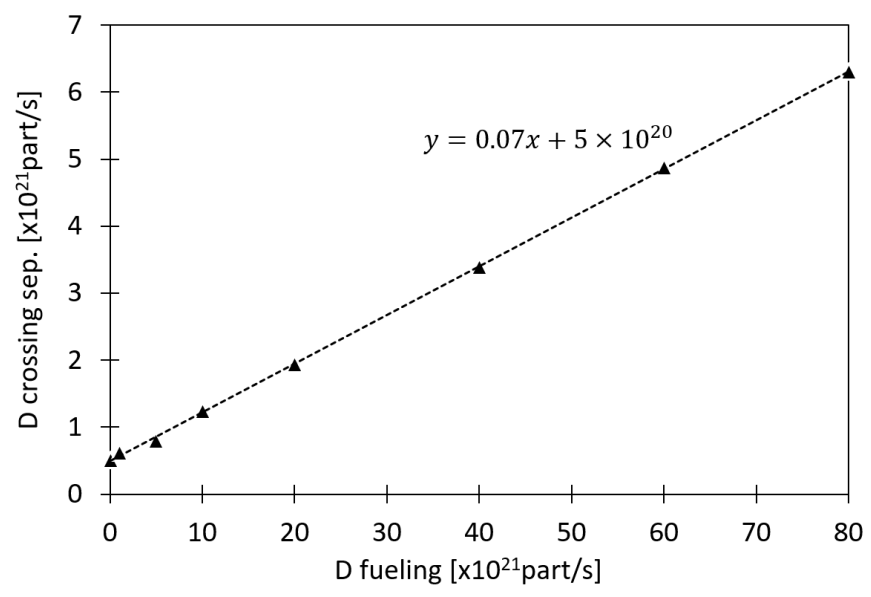

Figure 13: Dependence of neutral penetration across the separatrix on deuterium fuelling as derived from SOLEDGE2D-EIRENE simulations.

\subsubsection{Gyrokinetic simulations to validate QLK and TGLF for DTT full power parameters}

Linear and nonlinear gyrokinetic simulations have been performed at the fixed radius $\rho_{\text {tor }}=0.32$, using the fluxtube (radially local) version of the GENE code, in order to characterise the turbulence, compute the particle and heat fluxes and estimate the density peaking, testing the results of the ASTRA-TGLF and JINTRAC-QuaLiKiz predictive runs. This analysis has been carried out with the main goal of understanding which of the two transport simulations gives the more reliable estimate of the density peaking, since they give different results. The parameters from the end of the ASTRA-TGLF run have been used as GENE inputs. A detailed analysis of this case has been performed, and some parameters have been also replaced with the corresponding ones from the end of the JINTRAC-QuaLiKiz simulation, to investigate their impact on the results. The main simulation parameters (from ASTRA-TGLF) are summarised in Table3.

\begin{tabular}{|c|c|c|c|c|c|}
\hline$R / L_{n}$ & $R / L_{T e}$ & $R / L_{T i}$ & $T_{i} / T_{e}$ & $n_{A r} / n_{e}$ & $n_{W} / n_{e}$ \\
\hline 2.99 & 7.8 & 3.93 & 0.59 & $1.5 \mathrm{E}-3$ & $7.5 \mathrm{E}-5$ \\
\hline$q$ & $\hat{s}$ & $\kappa$ & $\delta$ & $\nu_{c}$ & $\beta_{e}$ \\
\hline 0.76 & 0.31 & 1.38 & $4.9 \mathrm{E}-2$ & $5.23 \mathrm{E}-5$ & $2.75 E-2$ \\
\hline
\end{tabular}

Table 3: Reference parameters from the end of the ASTRA-TGLF simulation, at the radius of analysis $\rho_{\text {tor }}=0.32$, used as GENE inputs.

Argon and Tungsten impurities with effective charge $Z_{\text {eff }}=1.65$ have been accounted for as kinetic species in the simulations when not differently stated. The normalised radial logarithmic gradients of the $f$ profiles $(f=n, T)$ are here defined as $R / L_{f}=-R d \ln f / d r$, where $R$ and $r$ are the plasma major and minor radii at the selected magnetic surface, respectively. The other parameters are the ion/electron temperature ratio $T_{i} / T_{e}$, the impurity density fractions $n_{\mathrm{imp}} / n_{e}$ (normalised with the electron density), the safety factor $q$, the magnetic shear $\hat{s}=(r / q) d q / d r$, the elongation $\kappa$, the triangularity $\delta$, the GENE collision parameter $\nu_{c}=2.3031 \times$ $10^{-5} \ln \Lambda R[m] n_{e}\left[10^{19} m^{-3}\right] / T_{e}[k e V]^{2}$, where $\ln \Lambda=24-$ $\ln \left(\sqrt{10^{13} n_{e}\left[10^{19} m^{-3}\right]} / 10^{3} T_{e}[k e V]\right)$ is the Coulomb logarithm, the ratio of the electron plasma pressure to the magnetic pressure $\beta_{e}=2 \mu_{0} n_{e} T_{e} / B_{0}$, with $\mu_{0}$ the vacuum permeability and $B_{0}=5.9 \mathrm{~T}$ the vacuum magnetic field on the magnetic axis. All the simulations are run with collisions, using a Landau operator. Since the electron-ion collision rate depends on $n_{i}$, which changes depending on the number of considered species $\left(n_{i}\right.$ is adapted using quasi-neutrality), the electron-ion thermal collision rate varies depending on the number of considered species and it can be easily evaluated for each case as $\nu_{e i}=4\left(n_{i} / n_{e}\right) \sqrt{T_{e} / m_{e}} \nu_{c} / R$. In the ASTRATGLF run the impurities have not been predicted, therefore their density profiles are set proportional to the electron (ion) one. A realistic geometry has been considered, with magnetic equilibrium obtained with the EFIT solver [73, 74], then approximated with a Miller analytic model [75]. Fast ions have been neglected due to their small density fraction $n_{F I} / n_{e} \sim 2 \%$ at the radius of analysis, but their potential effect on the transport should be investigated in the future. Finally, the effect of the $E \times B$ rotation shear has been neglected $\left(\gamma_{E}=-(r / q)\left(d \Omega_{\text {tor }} / d r\right) R / c_{s}=-0.03\right.$, where $\Omega_{\text {tor }}$ is the toroidal angular velocity and $c_{s} \equiv \sqrt{T_{e} / m_{i}}$ the ion sound speed), since its effect has been found to be negligible by performing a nonlinear GENE simulation at reference parameters.

To start, the linear $k_{y}$ spectra of the growth rate $\gamma$ and angular frequency $\omega$ corresponding to the first two most unstable modes have been computed with the GENE eigenvalue solver for reference parameters from the ASTRA-TGLF simulation at $\rho_{\text {tor }}=0.32$, in order to characterise the turbulence regime. The results are collected in Fig.14.

The growth rate $\gamma$ (a) and the angular frequency $\omega$ (b) are shown versus $k_{y}$, comparing simulations where the impurities have been neglected (black squares) with the ones where they have been taken into account (red triangles). $\gamma$ and $\omega$ are normalised with $c_{s} / R$, while $k_{y}$ is normalised with $1 / \rho_{s}$, where $\rho_{s}=c_{s} / \Omega_{i}$ is the sound Larmor radius, with $\Omega_{i}$ the ion cyclotron frequency. The dominant mode is a Trapped Electron Mode (TEM) at all the wavenumbers, while the second unstable mode is an Ion Temperature Gradient (ITG) mode, corresponding to $\omega<0$ and $\omega>0$ respectively, according to GENE conventions. The sub-dominant ITGs are peaked at a larger wavenumber $\left(k_{y, \text { ITG peak }} \rho_{s} \sim 0.6\right)$ compared to the dominant TEMs $\left(k_{y}\right.$, TEM peak $\left.\rho_{s} \sim 0.4\right)$. As a consequence the ITGs are even more non-linearly sub-dominant, since 

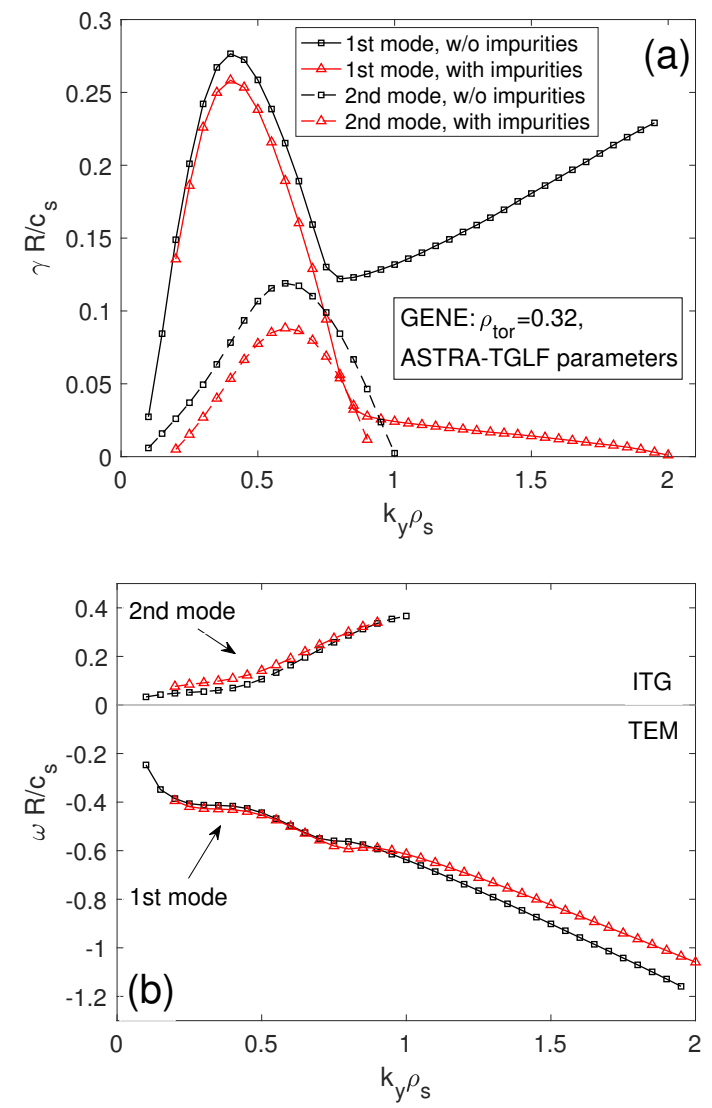

Figure 14: (color online) $k_{y}$ spectra of the growth rate $\gamma$ (a) and angular frequency $\omega$ (b) of the most unstable linear mode (solid) and of the second unstable linear mode (dashed), neglecting (black) or taking into account (red) the impurities, for ASTRA-TGLF parameters at $\rho_{\text {tor }}=$ 0.32. Both $\gamma$ and $\omega$ are normalised with $c_{s} / R$, while $k_{y}$ is normalised with $1 / \rho_{s}$.

smaller wavenumbers most contribute to the NL fluxes. Micro Tearing Modes (MTM) are found with the GENE initial value solver for $k_{y} \rho_{s} \leq 0.1$, which are destabilised by the finite $\beta_{e}$ (they are identified as MTMs looking at the ballooning structures of the electrostatic potential fluctuation $\delta \phi$ and of the parallel vector potential fluctuation $\left.\delta A_{\|}\right)$. However, they correspond to small growth rates and do not impact the nonlinear (NL) fluxes at reference parameters. Finally, the impurities have a small stabilising effect on the smaller $k_{y}$ TEM branch, a moderate effect on the subdominant ITGs, while they considerably stabilise TEMs at $k_{y} \rho_{s}>0.8$, as seen comparing the red and black curves in Fig.15 (a).

As a second step, a quasi-linear (QL) evaluation of the electron particle flux $\Gamma_{e}$ spectrum dependence on $R / L_{n}$ has been pursued using a simple ES 'mixing length' model based on GENE linear simulations, following [76, 77]. For each $k_{y}$, the $\mathrm{QL}$ fluxes are evaluated as $F^{Q L}=$ $A_{0} \sum_{k_{y}} w^{Q L}\left(k_{y}\right) F_{\text {norm }}^{L}\left(k_{y}\right)$, where $A_{0}$ is a scaling factor
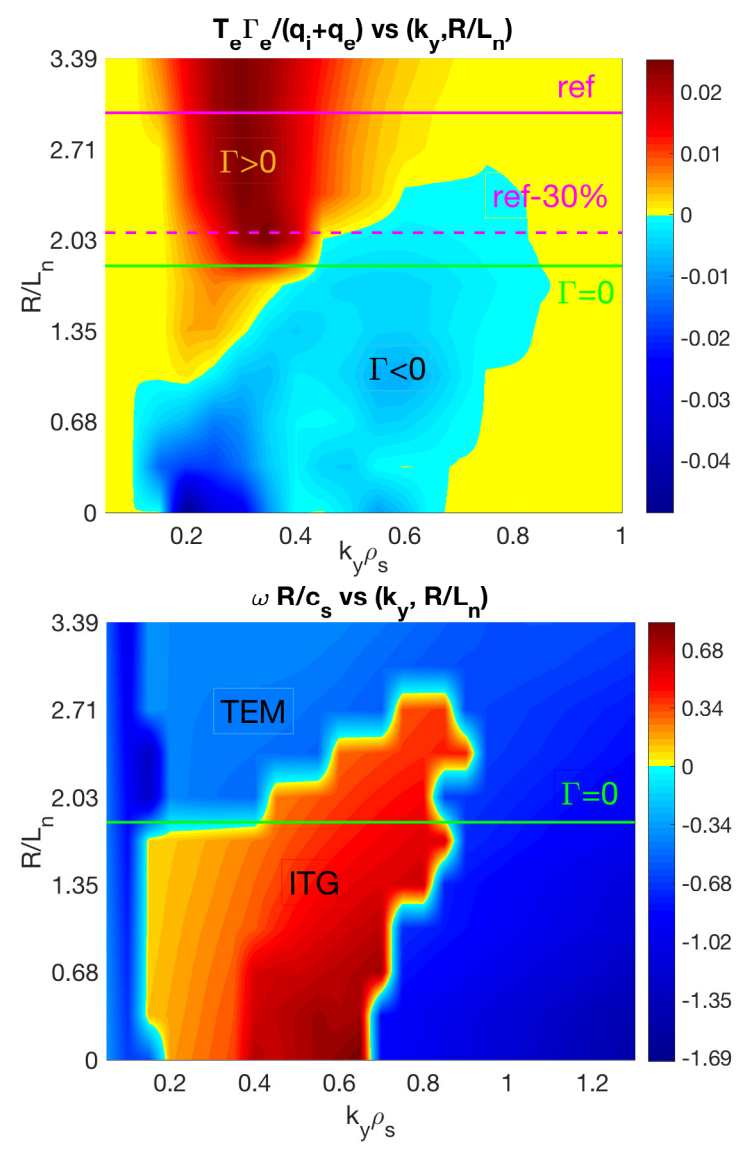

Figure 15: (color online) (a) Spectrum of the QL electron particle flux $\Gamma_{e}\left(k_{y}\right)$ versus $R / L_{n}$, normalised with $\left(q_{e}+\right.$ $\left.q_{i}\right) / T_{e}$, where $q_{e}$ and $q_{i}$ are the total QL electron an ion heat fluxes and $\Gamma_{e}\left(k_{y}\right)$ satisfies $\Gamma_{e}=\sum_{k_{y}} \Gamma_{e}\left(k_{y}\right)$

- (b) $k_{y}$ spectrum of the growth rate $\gamma$ of the most unstable linear mode versus $R / L_{n} . \gamma$ is normalised with $c_{s} / R$, while $k_{y}$ is normalised with $1 / \rho_{s}$.

associated to the absolute fluctuation amplitude, which is the same for different fluxes $F=\Gamma_{e}, q_{e}, q_{i}$ and cancels out when computing flux ratios, $F_{\text {norm }}^{L}\left(k_{y}\right)$ represents a properly normalised spectral contribution to the flux which is evaluated with the fields from the corresponding linear eigenmode, and the QL saturation prescriptions $w^{Q L}\left(k_{y}\right)=\left(\gamma /\left\langle k_{\perp}^{2}\right\rangle\right)^{\xi}$ specify the $k_{y}$ dependence of the relative saturation amplitude levels of the NL electrostatic potential $\phi$. Here $\left\langle k_{\perp}^{2}\right\rangle$ indicates the fluxsurface average of the squared perpendicular wave number, weighted with the $|\phi|^{2}$ ballooning structure, considering only $k_{x}=-\Delta k_{x}, 0, \Delta k_{x}\left(\Delta k_{x}=2 \pi / L_{x}\right.$, with $L_{x}$ the $\mathrm{x}$ box size) following [78], and setting $\xi=2$ (this, a posteriori, gives the best QL-NL spectra agreement). The QL results are summarised in Fig.15.

Figure15 (a) shows the $\Gamma_{e}$ spectrum, normalised with the total (summed over $k_{y}$ ) value of $\left(q_{e}+q_{i}\right) / T_{e}$, in the $\left(k_{y} \rho_{s}, R / L_{n}\right)$ plane. The 'zero particle flux' condition $\Gamma_{e}=0$ (green line), which is very close to the ac- 
tual $\Gamma_{e}=\Gamma_{e, r e f}$. from ASTRA-TGLF inputs $\left(\Gamma_{e, r e f .} \sim\right.$ $1.01 \times 10^{18} \mathrm{~s}^{-1} \mathrm{~m}^{-2}$ at the radius of analysis, therefore it is almost negligible), is satisfied for $R / L_{n} \sim 1.8$, which is smaller than the TGLF SAT1-geo reference value $R / L_{n} \sim 3$ (magenta solid line), below a $\pm 30 \%$ error bar. The $\Gamma_{e}=0$ condition is obtained from a balance of low$\mathrm{k}$ TEM-driven outward flux with a larger-k ITG-driven inward flux. This correspondence of the TEM and ITG regimes with the $\Gamma_{e}$ signs is obtained comparing Fig.15 (a) with Fig.15 (b), which shows the frequency $\omega$ in the $\left(k_{y} \rho_{s}, R / L_{n}\right)$ plane $(>0$ for ITG and $<0$ for TEM according to GENE conventions).

$R / L_{n}$ scans of NL GENE ion-scale local runs have been performed to obtain a GK estimate of the peaking (i.e. the $R / L_{n}$ that satisfies $\Gamma_{e} \sim 0$ ). The GENE results are shown in Fig.16 by solid black lines, compared with the ones that are obtained running TGLF SAT1-geo (solid/red) and TGLF SAT2 (solid/blue) stand-alone simulations.

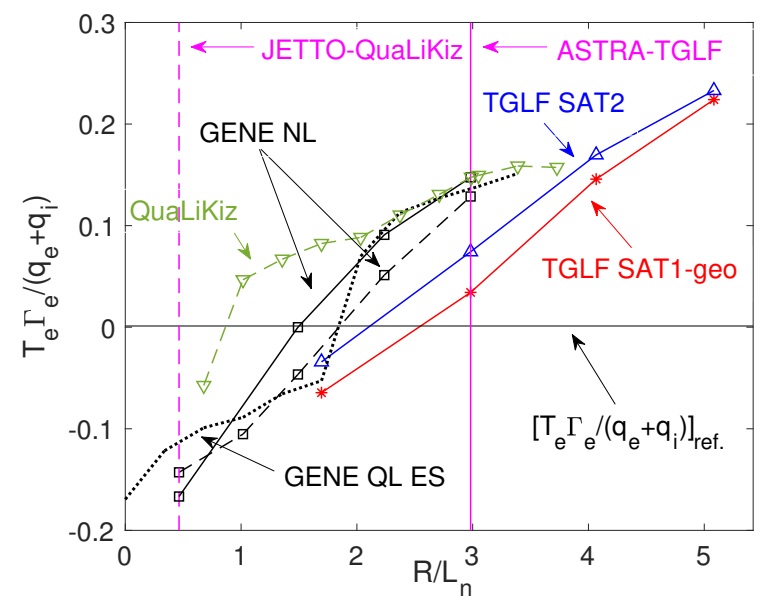

Figure 16: (color online) Normalised electron particle flux $T_{e} \Gamma_{e} /\left(q_{e}+q_{i}\right)$ vs $R / L_{n}$, with parameters from ASTRA-TGLF (solid or dotted) and JINTRACQuaLiKiz (dashed) predictive transport simulations, computed with GENE NL (black), GENE QL (black, dotted), TGLF SAT1-geo (red), TGLF SAT2 (blue) and QuaLiKiz (green), where the QL codes have been run using the stand-alone version. The reference values $R / L_{n} \sim 3$ (ASTRA-TGLF) and $R / L_{n}=0.46$ (JINTRAC-QuaLiKiz) are shown by solid and dashed vertical magenta lines, respectively.

An additional $R / L_{n}$ scan of GENE NL runs (dashed/black) has been performed replacing the values of the temperature logarithmic gradients $R / L_{T e}=7.8$, $R / L_{T i}=3.93$ (ASTRA-TGLF) with the ones corresponding to the JINTRAC-QuaLiKiz predictive simulation: $R / L_{T e}=9.08, R / L_{T i}=4.58$, compared with corresponding QuaLiKiz stand-alone simulations (dashed/green). The reference value of $R / L_{n} \sim 3$ from
ASTRA-TGLF is shown by a solid vertical magenta line, while the corresponding value $R / L_{n}=0.46$ from JINTRAC-QuaLiKiz by a dashed vertical magenta line. More in detail, Fig.16 shows the normalised electron particle flux $T_{e} \Gamma_{e} /\left(q_{i}+q_{e}\right)$ vs $R / L_{n}$. The predicted density peaking by each code is obtained from Fig.16 (a) at the crossing of the corresponding curve with the horizontal line $\Gamma_{e} \sim 0$. It follows that GENE prediction $\left(R / L_{n} \sim 1.4-1.8\right)$ lies in between the QuaLiKiz and TGLF ones, not confirming the 'flat $n_{e}$ ' prediction of QuaLiKiz. In particular, even replacing the ASTRA-TGLF $R / L_{T e, i}$ values with those from JINTRAC-QuaLiKiz, GENE still predicts a peaked $n_{e}$, with even slightly larger $R / L_{n}$.

The sensitivity of the GENE estimate of the electron density peaking to changes in $R / L_{T e}$, which is the main driver of the TEM-dominant turbulence regime, has been tested by repeating the nonlinear $R / L_{n}$ scans, increasing and decreasing the reference ASTRA-TGLF value $R / L_{T e}=7.8$ by $\pm 20 \%$. The results, shown in Fig.17, indicate that the effect of changing $R / L_{T e}$ within a $\pm 20 \%$ error bar has a small/moderate effect on the peaking, which increases by $\sim 20 \%$ when $R / L_{T e}$ is decreased.

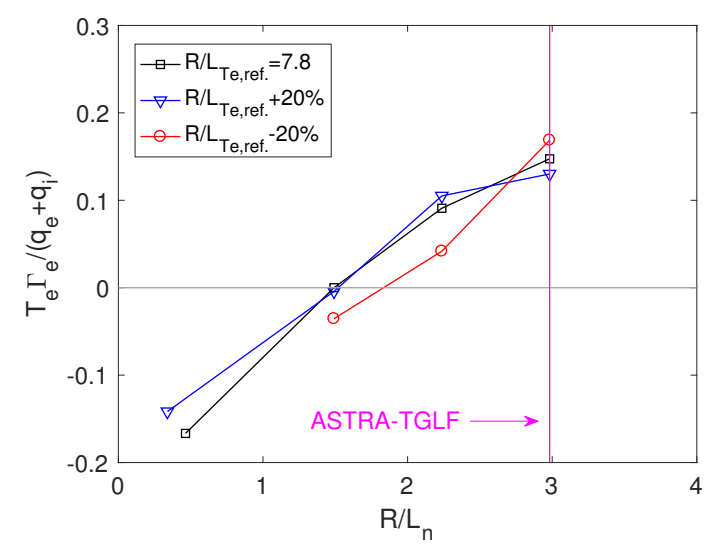

Figure 17: (color online) Normalised electron particle flux $T_{e} \Gamma_{e} /\left(q_{e}+q_{i}\right)$ vs $R / L_{n}$, for the three values $R / L_{T e}=7.8,7.8 \pm 20 \%$ of the normalised electron temperature logarithmic gradient.

However, a similar sensitivity test in QuaLiKiz indicated a significant increase in zero-particle-flux R/Lne with decreasing $R / L_{T e}$. Zero-particle-flux at $R / L_{n e}=1.5$ (the GENE value) was attained with only a $\sim 15 \%$ decrease in $R / L_{T e}$. The zero-flux boundary in QuaLikiz is extremely sensitive to the ITG-TEM transition in this regime, and occurs here rapidly over multiple wavenumbers with stiff TEM heat flux, also meaning that the ad-hoc TEM electron heat flux model tested here was less effective than expected in reducing the power-balance $R / L_{T e}$. The selforganized $R / L_{n}-R / L_{T}$ state that leads to flat ne profiles in the QuaLiKiz simulations for $\rho_{\text {tor }}<0.4$ in this specific regime thus likely arises from discrepancies in the 
ITG-TEM instability boundary in $R / L_{n}-R / L_{T}$ space compared to higher-fidelity gyrokinetic models. This will be explored in future work.

Summing up, the very flat profile predicted by JINTRAC-QuaLiKiz in the inner region is not validated by a comparison of QuaLiKiz stand-alone with GENE. Also, the amount of $n_{e}$ peaking predicted by ASTRATGLF turns out to be a bit overestimated when compared with GENE, although qualitatively nearer to the gyrokinetic prediction. Both models should then be taken with care in the region inside $\rho_{\text {tor }}=0.4$, which is characterised by high power density, TEM dominance and $q$ values below 1 , with sawteeth not yet accounted for.

\subsection{FP scenario heating mixes}

Prior to this modelling work, the heating mix of the FP scenario was not established. One of the purposes of this work was to optimise the choice of power distribution amongst the 3 systems and of the NBI energy. In addition to the three heating mix options proposed in [6], other possible candidates have been suggested within the DTT physics group. The various options are listed in table 4 . In order to assist the heating mix choice, each of these $9 \mathrm{SN}$ full power $\mathrm{H}$-mode scenarios has been simulated both in a JETTO run with the standard QLK model and with a JINTRAC-ASTRA approach with TGLF SAT1geo model.

For the sake of clarity, some radial profiles of only the 3 most salient options, obtained from TGLF SAT1-geo runs are shown in figure 18. Main parameters of these 3 cases are displayed in table 5 . In all the FP options, the characteristic behaviour of the main plasma profiles seen in sect. 3.5.1 recurs. Particularly, the electron density has a moderately peaked profile reaching maximum values in the range of $(1.9-2.7) \times 10^{20} \mathrm{~m}^{-3}$ and in the central plasma region the electron temperature $T_{e} \approx 15-27 \mathrm{keV}$ is much higher than the ion temperature $T_{i} \approx 8-12 \mathrm{keV}$.

In order to achieve more central NBI deposition as well as to minimise the collisionless ripple fast particle losses[79] and allow resonant excitation of Alfvénic waves[80], the higher NBI energy has been preferred to the higher NBI power option, in addition to opting for the largest possible injection angle.

Given the need of trying to equilibrate $T_{e}$ and $T_{i}$, the option B with $40 \mathrm{MW}$ of ECH power has been discarded, and the missing NBI power has been replaced by ICH power, which has the double advantage of providing central ion heating and a fast particle population that could help lowering the high ion stiffness observed by TGLF and QLK $[60]$. From the power deposition calculations, the synergy effects between ICRH and NBI proved to be very relevant, leading to a maximised energetic particle content in option D.

With the state-of-the-art in quasi-linear models, differences between the various options are not large. However,

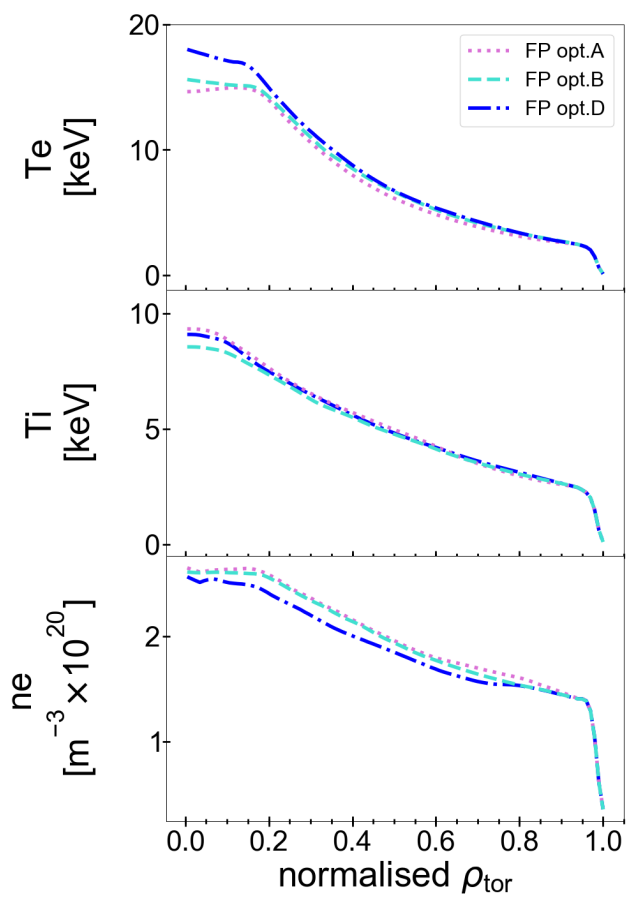

(a)

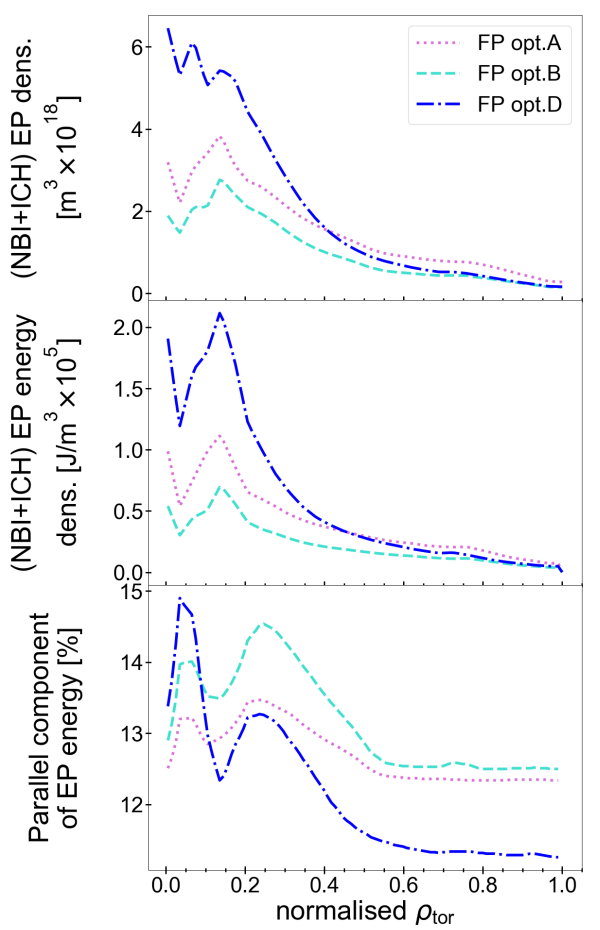

(b)

Figure 18: Comparison of radial profiles among the most salient options of the FP scenario. (a) Profiles of electron temperatures, ion temperatures, and electron densities. (b) Profiles of energetic particle densities, energetic particle energy densities, and parallel components of the energetic particle energies. 
the non-linear effects linked with thermal and suprathermal pressure gradients not included in the QL models could play an important role on the ion temperature profile.

From the physics point of view, the option D is the best compromise between technical feasibility, need to heat ions, and creation of suitable EP population.

\subsection{Day 1 scenario}

In the day- 1 phase (with $B_{t}=6 \mathrm{~T}$ and $I_{p l}=4.0 \mathrm{MA}$ ), the power coupled to the plasma $(\sim 25 \mathrm{MW})$ will be shared among the heating systems as described in the sect. 2. The integrated modelling of a steady-state Deuterium plasma in the day-1 scenario has been performed using both the standard QLK model in a JETTO run and the TGLF SAT1-geo model with the JINTRAC-ASTRA approach.

The simulation settings widely described from the beginning of sect. 3.1 up to the end of sect. 3.4 has been also employed in these day-1 scenario modelling work.

Since the density value at the top of the pedestal has not been reduced with respect to the full power case, the Greenwald fraction increased to $\langle n e\rangle \sim 0.5 n_{G}$, still well within safety margins. The pedestal parameters predicted by Europed for day-1 scenario are very similar to the full power case ones.

The electron temperature $T_{e}$, ion temperature $T_{i}$, electron density $n_{e}$, toroidal rotation $\omega_{\text {tor }}$, and safety factor $q$ radial profiles obtained by TGLF SAT1-geo and QLK runs are displayed in figure 19. According both models, the density peaking results less pronounced in day-1 phase than in the reference FP scenario, leading to a lower central value $n_{e_{0}} \approx 2.0 \times 10^{20} / \mathrm{m}^{3}$.

We notice some discrepancies between QLK and TGLF temperature profiles. In the day-1 case, the electron temperature is estimated rather similar in the QLK run with respect to the full power case, while is significantly reduced in the TGLF run. For both models, the $T_{i}$ values are similar in day-1 and FP scenarios, in spite of having half the injected power. This may be ascribed both to the increased ITG threshold with lower $T_{e} / T_{i}$ and to the high ion stiffness, which makes a factor 2 difference in power rather ineffective in terms of $T_{i}$ profiles (although crucial for divertor studies).

In figure 20(a), there are impurity densities and effective charge radial profiles for the standard QLK run (solid lines) compared to an assessment of the impurity and $Z_{\text {eff }}$ profiles in presence of TGLF SAT1-geo predicted profiles carried out with JETTO/SANCO and QLK (dashed lines). We note that the impurities feature less central accumulation than in the full power case.

For the TGLF case, shown in figure 20(b), the neutron rate is reduced to $7.1 \mathrm{e} 16$ neutrons/s. In figure $20(\mathrm{c})$ the EP contents are shown for the TGLF SAT1-geo case.

In figure $21(\mathrm{a})$ the radial profiles of all power densities

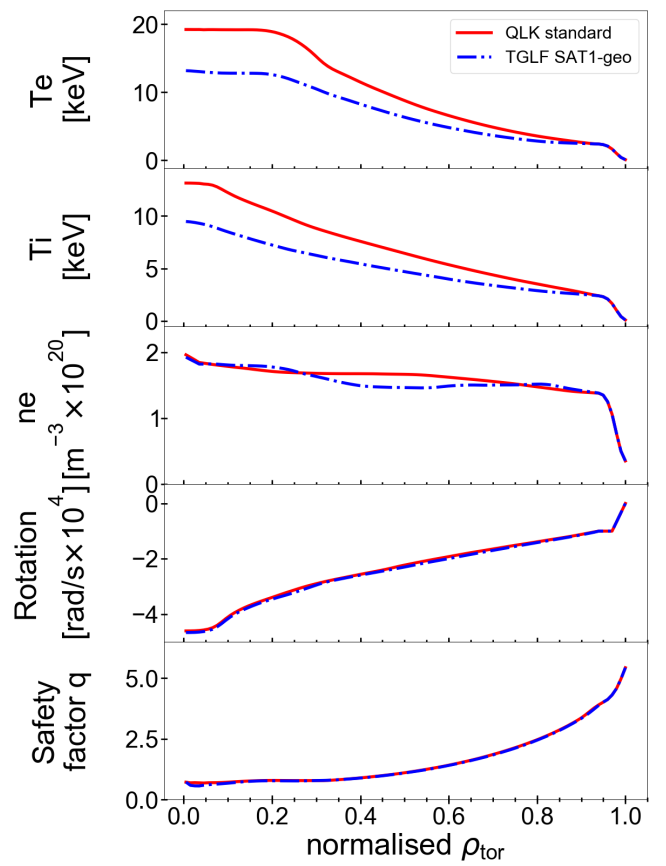

Figure 19: Steady-state radial profiles of the electron and ion temperatures, electron density, toroidal rotation, and safety factor of the day-1 scenario, with turbulent transport calculated by TGLF SAT1-geo (blue dash-dot line).

and those of the total electron and ion powers are shown in figure 21 (b), only for the standard QLK simulation.

\subsection{Day 0 scenario}

The day- 0 phase (with $B_{t}=3 \mathrm{~T}$ and $I_{p l}=2.0 \mathrm{MA}$ ) features only $8 \mathrm{MW}$ of $\mathrm{ECH}$ power in second harmonic $\mathrm{X}$-mode (coupling to the plasma $\sim 7.2 \mathrm{MW}$ of power), as described in sect. 2. The integrated modelling of a steady-state Deuterium plasma in the day-0 scenario has been performed using the TGLF SAT1-geo model with the JINTRAC-ASTRA approach. We do not show QLK results for this case, as this purely electron heated case is the one where QLK validity is most affected by the dominant TEM regime.

The simulation settings widely described in the initial sections of 3 has been also used in this day-0 scenario modelling work. While maintaining the same relative impurity mix of Ar and W used in FP runs, in the day-0 simulation has been set a flat $Z_{\text {eff }}=1.4$ profile as initial condition in SANCO. The density at the pedestal top has been reduced with respect to the full power case, to have approximately a Greenwald fraction of about 0.36 .

In figure 22(a), the radial profiles of $T_{e}, T_{i}, n_{e}$, and $q$ predicted by the TGLF SAT1-geo run of day- 0 phase are shown. The reduced pedestal density with respect to the full power case and the slightly peaked $n_{e}$ profile lead to a 
Table 4: Heating mix options (power values at plasma) evaluated for DTT full power scenarios.

\begin{tabular}{|c|c|c|c|}
\hline Option & ECRH & $\mathrm{ICRH}$ & NBI \\
\hline $\mathrm{A}$ & $\begin{array}{c}\sim 28.8 \mathrm{MW} \\
(32 \text { gyr. } \times 1 \mathrm{MW}) \times 0.9\end{array}$ & $\begin{array}{c}\sim 3 \mathrm{MW} \\
(2 \text { antennas in } 1 \text { module })\end{array}$ & $\begin{array}{c}\sim 15 \mathrm{MW} \\
2 \mathrm{inj} . \times(7.5 \mathrm{MW} \text { at } 400 \mathrm{keV})\end{array}$ \\
\hline B & $\begin{array}{c}\sim 36.0 \mathrm{MW} \\
(16 \text { gyr. } \times 1 \mathrm{MW}+20 \text { gyr. } \times 1.2 \mathrm{MW}) \times 0.9\end{array}$ & $\begin{array}{c}\sim 3 \mathrm{MW} \\
(2 \text { antennas in } 1 \text { module })\end{array}$ & $\begin{array}{c}\sim 7.5 \mathrm{MW} \\
1 \mathrm{inj.} \times(7.5 \mathrm{MW} \text { at } 400 \mathrm{keV})\end{array}$ \\
\hline $\mathrm{C}$ & $\begin{array}{c}\sim 28.8 \mathrm{MW} \\
(32 \text { gyr. } \times 1 \mathrm{MW}) \times 0.9\end{array}$ & $\begin{array}{c}\sim 9 \mathrm{MW} \\
(6 \text { antennas in } 3 \text { modules })\end{array}$ & $\begin{array}{c}\sim 7.5 \mathrm{MW} \\
1 \mathrm{inj.} \times(7.5 \mathrm{MW} \text { at } 400 \mathrm{keV})\end{array}$ \\
\hline $\mathrm{D}$ & $\begin{array}{c}\sim 30.2 \mathrm{MW} \\
(24 \text { gyr. } \times 1 \mathrm{MW}+8 \text { gyr. } \times 1.2 \mathrm{MW}) \times 0.9\end{array}$ & $\begin{array}{c}\sim 6 \mathrm{MW} \\
(4 \text { antennas in } 2 \text { modules })\end{array}$ & $\begin{array}{c}\sim 10 \mathrm{MW} \\
1 \mathrm{inj.} \times(10 \mathrm{MW} \text { at } 500 \mathrm{keV})\end{array}$ \\
\hline $\mathrm{E}$ & $\begin{array}{c}\sim 28.8 \mathrm{MW} \\
(32 \text { gyr. } \times 1 \mathrm{MW}) \times 0.9\end{array}$ & $\begin{array}{c}\sim 3 \mathrm{MW} \\
(2 \text { antennas in } 1 \text { module })\end{array}$ & $\begin{array}{c}\sim 15 \mathrm{MW} \\
1 \mathrm{inj} . \times(10 \mathrm{MW} \text { at } 500 \mathrm{keV})+ \\
+1 \mathrm{inj} . \times(5.0 \mathrm{MW} \text { at } 200 \mathrm{keV})\end{array}$ \\
\hline $\mathrm{F}$ & $\begin{array}{c}\sim 28.8 \mathrm{MW} \\
(32 \text { gyr. } \times 1 \mathrm{MW}) \times 0.9\end{array}$ & $\begin{array}{c}\sim 3 \mathrm{MW} \\
(2 \text { antennas in } 1 \text { module })\end{array}$ & $\begin{array}{c}\sim 10 \mathrm{MW} \\
1 \mathrm{inj} . \times(10 \mathrm{MW} \text { at } 600 \mathrm{keV})\end{array}$ \\
\hline $\mathrm{G}$ & $\begin{array}{c}\sim 30.2 \mathrm{MW} \\
(24 \text { gyr. } \times 1 \mathrm{MW}+8 \text { gyr. } \times 1.2 \mathrm{MW}) \times 0.9\end{array}$ & $\begin{array}{c}\sim 6 \mathrm{MW} \\
(4 \text { antennas in } 2 \text { modules })\end{array}$ & $\begin{array}{c}\sim 7.5 \mathrm{MW} \\
1 \mathrm{inj} . \times(7.5 \mathrm{MW} \text { at } 400 \mathrm{keV})\end{array}$ \\
\hline $\mathrm{H}$ & $\begin{array}{c}\sim 30.2 \mathrm{MW} \\
(24 \text { gyr. } \times 1 \mathrm{MW}+8 \text { gyr. } \times 1.2 \mathrm{MW}) \times 0.9\end{array}$ & $\begin{array}{c}\sim 6 \mathrm{MW} \\
(4 \text { antennas in } 2 \text { modules })\end{array}$ & $\begin{array}{c}\sim 10 \mathrm{MW} \\
1 \mathrm{inj.} \times(10 \mathrm{MW} \text { at } 400 \mathrm{keV})\end{array}$ \\
\hline I & $\begin{array}{c}\sim 26.3 \mathrm{MW} \\
(16 \text { gyr. } \times 1 \mathrm{MW}+11 \text { gyr. } \times 1.2 \mathrm{MW}) \times 0.9\end{array}$ & $\begin{array}{c}\sim 9 \mathrm{MW} \\
(6 \text { antennas in } 3 \text { modules })\end{array}$ & $\begin{array}{c}\sim 10 \mathrm{MW} \\
1 \mathrm{inj} . \times(10 \mathrm{MW} \text { at } 500 \mathrm{keV})\end{array}$ \\
\hline
\end{tabular}

Table 5: Main parameters of a sub-set of heating options for the DTT FP scenario using TGLF SAT1-geo.

\begin{tabular}{ccccccccc}
\hline $\begin{array}{c}\text { Heating } \\
\text { Option }\end{array}$ & $\begin{array}{c}\tau_{E}[\mathrm{~s}] \\
\left(P=P_{\text {tot }}\right)\end{array}$ & $\begin{array}{c}\tau_{E}[\mathrm{~s}] \\
\left(P=P_{\text {sep }}\right)\end{array}$ & H98Y & $\beta_{\mathrm{N}_{\text {therm }}} / \beta_{\mathrm{N}_{\text {tot }}}$ & $\begin{array}{c}W_{\mathrm{EP}} / W_{\text {tot }} \\
{[\%]}\end{array}$ & $\begin{array}{c}T_{e_{0}} / T_{i_{0}} \\
{[\mathrm{keV} / \mathrm{keV}]}\end{array}$ & $\left\langle n_{e}\right\rangle / n_{G}$ & $\begin{array}{c}\text { Neutron } \\
\text { rate }\end{array}$ \\
\hline $\mathrm{A}$ & 0.25 & 0.45 & 0.95 & $1.40 / 1.49$ & 5.7 & $14.6 / 9.3$ & 0.47 & $1.24 \times 10^{17} \mathrm{~s}$ \\
\hline $\mathrm{B}$ & 0.26 & 0.44 & 0.95 & $1.42 / 1.46$ & 3.3 & $15.6 / 8.5$ & 0.46 & $0.85 \times 10^{17} \mathrm{~s}$ \\
\hline $\mathrm{D}$ & 0.25 & 0.43 & 0.95 & $1.39 / 1.49$ & 6.5 & $18.0 / 9.1$ & 0.44 & $1.29 \times 10^{17} \mathrm{~s}$ \\
\hline
\end{tabular}




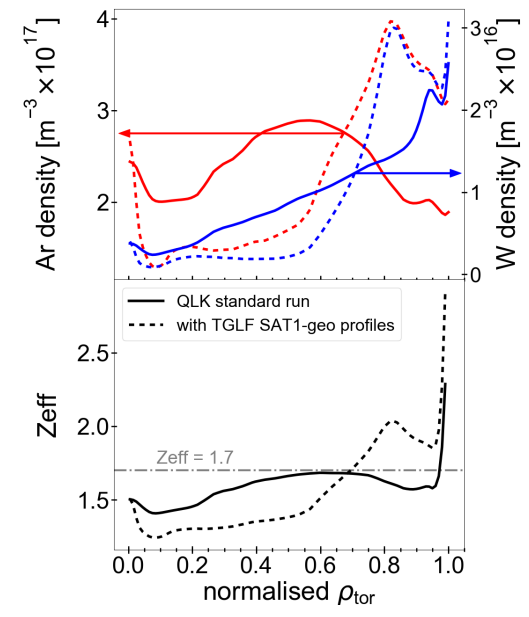

(a)

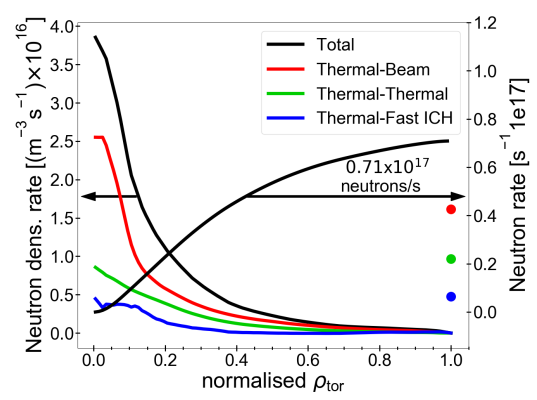

(b)

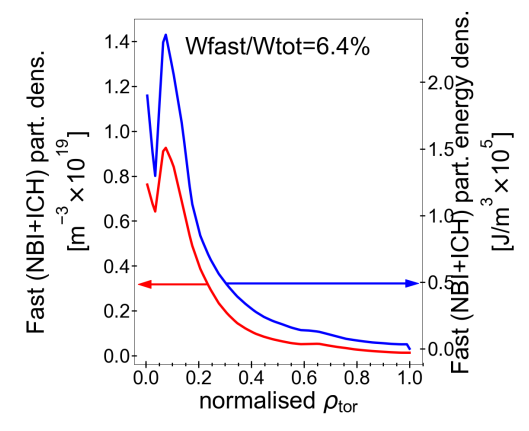

(c)

Figure 20: (a) Radial profiles of the impurity densities (Ar in red, $\mathrm{W}$ in blue) and of effective charge in the day- 1 scenario for the TGLF SAT1-geo case. The standard QLK case results (solid lines) are compared to an assessment of the impurity and $Z_{\text {eff }}$ profiles in presence of TGLF SAT1geo predicted profiles carried out with JETTO/SANCO and QLK (dashed lines). (b) Radial profiles of neutron density rates (neutrons from thermal nuclei in green, from a thermal nucleus and a fast NBI nucleus in red, and from a thermal nucleus and a fast ICRH nucleus in blue). The radial profile of the total neutron rate is also displayed (black). (c) Radial profiles of density and energy density of EPs due to the both NBI and ICRH systems.

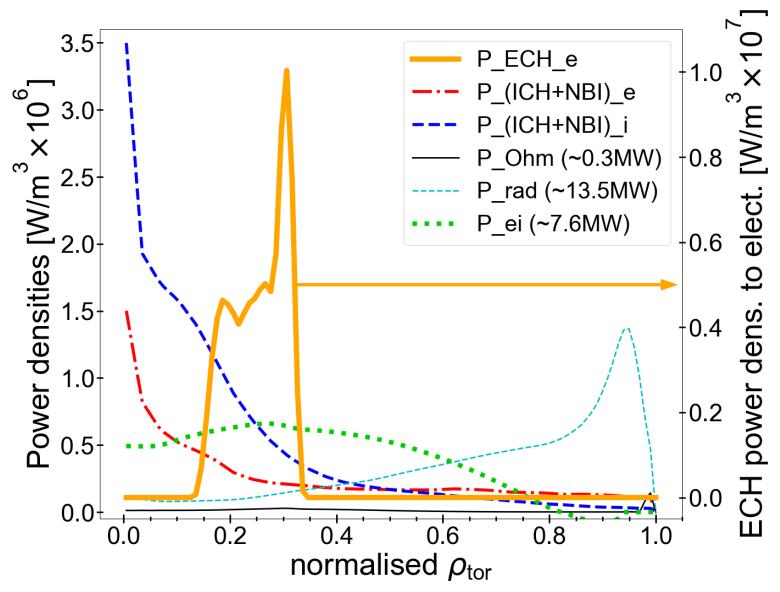

(a)

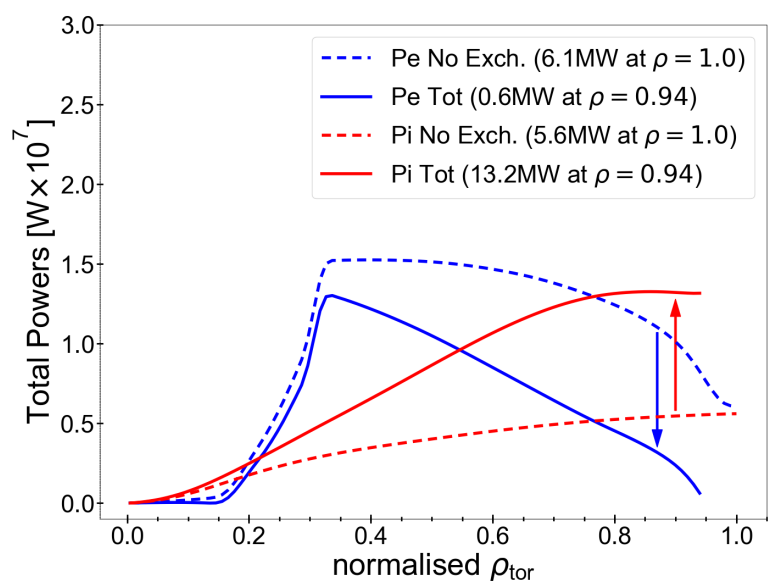

(b)

Figure 21: (a) Radial profiles of power densities in the day-1 scenario: ECRH power deposited to electrons $P_{\mathrm{ECH} e}, \mathrm{NBI}$ and ICRH power deposited to electrons $P_{(\mathrm{ICH}+\mathrm{NBI}) e}, \mathrm{NBI}$ and ICRH power deposited to ions $P_{(\mathrm{ICH}+\mathrm{NBI})}$, Ohmic power $P_{\mathrm{Ohm}}$, radiative power $P_{\mathrm{rad}}$, and thermal exchange power between electrons and ions $P_{e i}$. (b) Radial profiles of electron and ion total powers including or not including the thermal exchange power between species in the day- 1 scenario. 


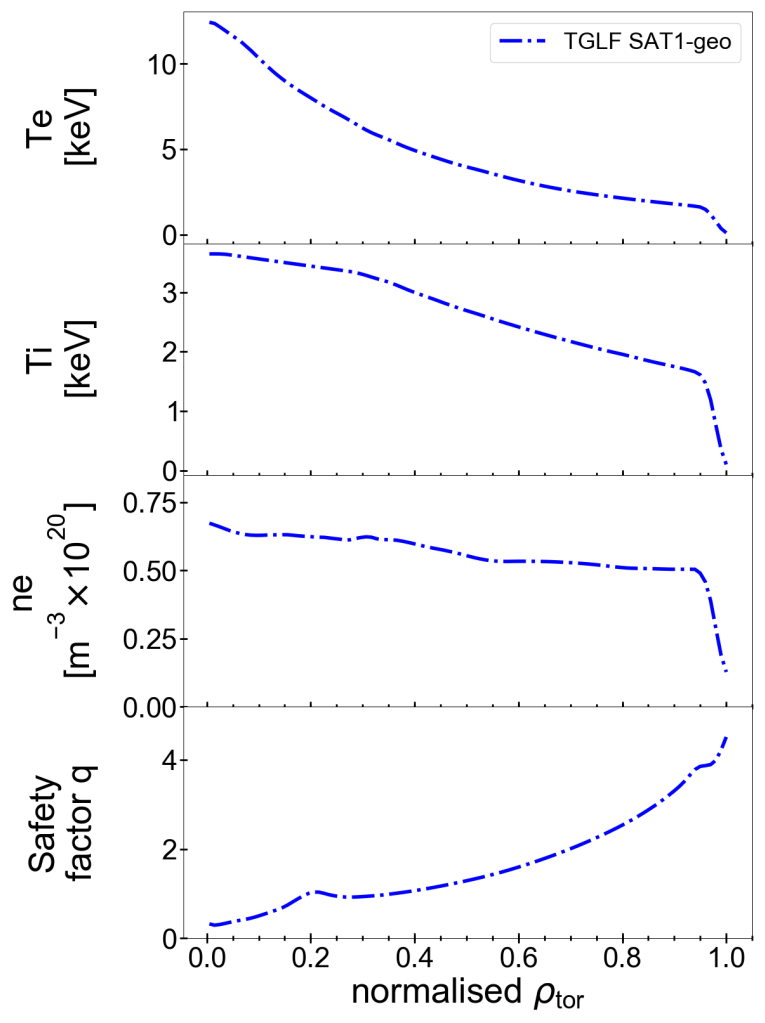

(a)

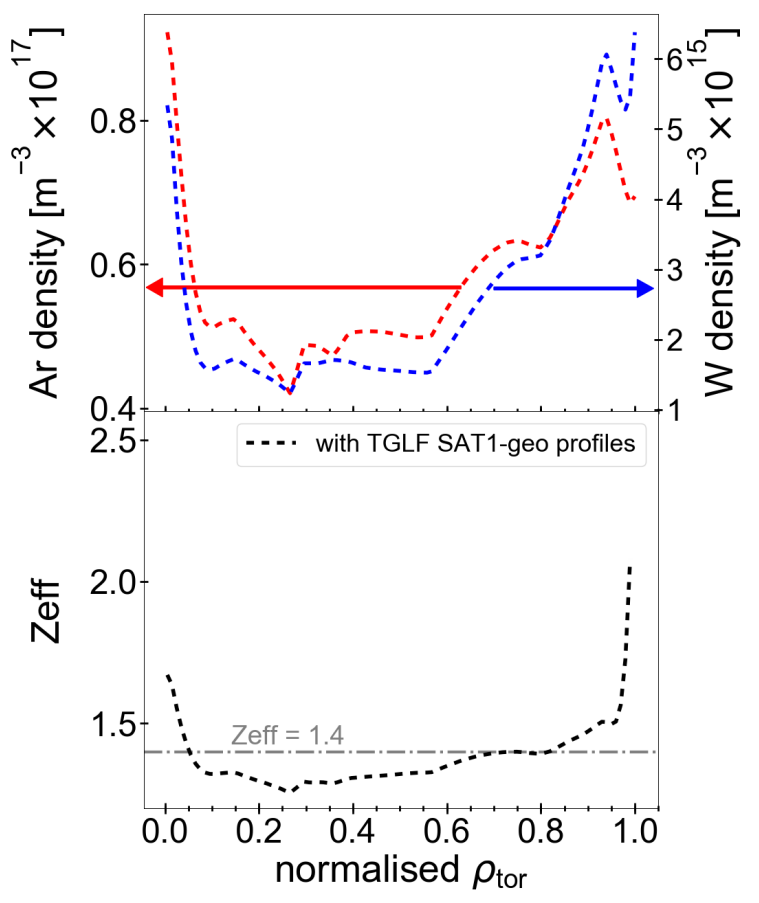

(b)

Figure 22: (a) Steady-state radial profiles of the electron and ion temperatures, electron density, and safety factor of the day-0 scenario, with turbulent transport calculated by TGLF SAT1-geo (blue dash-dot line). (b) Radial profiles of the impurity densities (Argon in red, Tungsten in blue) and of effective charge $Z_{\mathrm{eff}}$ in the day-0 scenario in presence of TGLF SAT1-geo predicted profiles carried out with JETTO/SANCO and QLK (dashed lines) 
lower central density value of about $n_{e_{0}} \approx 0.7 \times 10^{20} / \mathrm{m}^{3}$.

In figure 22(b), impurity densities and effective charge radial profiles are shown for an assessment of the impurity and $Z_{\text {eff }}$ profiles in presence of TGLF SAT1-geo predicted profiles carried out with JETTO/SANCO and QLK (dashed lines). $T_{e}$ is much larger that $T_{i}\left(T_{e_{0}} \approx\right.$ $12 \mathrm{keV}$ and $T_{i_{0}} \approx 4 \mathrm{keV}$ ), due to having only electron heating and low density. The toroidal rotation is not shown as there is no NBI torque, so it reduces to intrinsic rotation, with edge values difficult to estimate but expected small. We note that the impurities show much less core penetration in the day-0 case.

For the sake of completeness, in figure 21 the radial profiles of all power densities and those of the total electron and ion powers are shown in figure 23 (a) and 23 (b) respectively.

A neutron rate of $\sim 4 \times 10^{14}$ neutrons/s has been estimated for the day- 0 scenario.

\section{Conclusions}

The first-principle multi-channel integrated modelling of the main DTT baseline scenarios using quasi-linear transport models (TGLF, QLK) has started and is key to support the design of the device and to help the elaboration of a DTT scientific work-programme. Particularly, this work has been crucial to define the reference heating mix for the full power scenario among 9 possible options. Moreover, results from this modelling led to the decision to enlarge the device up to $R_{0}=2.19 \mathrm{~m}$ and $a=0.70 \mathrm{~m}$. Reference profiles in different scenarios are now available for diagnostic system design, as well as estimates of neutron yields and fast particle losses. In addition, a preliminary risk evaluation of a fuelling via gas puffing without the pellet support has been done, suggesting a beneficial impact of incorporating pellet fuelling in addition to gas puff. Some validation of the quasi-linear models used against gyrokinetic simulations in the specific DTT range of parameters has been performed, as an essential procedure to improve the reliability of such predictions.

\section{Acknowledgements}

This work is carried out in the frame of the DTT design activity. The authors are very grateful to all the colleagues involved in the DTT project for their precious contribution. We thank G. Tardini for providing help for the simulations with ASTRA code. We thank G. M. Staebler for providing advice on the use of the TGLF model and T. Goerler for advice on the GENE simulations. This work was part-funded by the RCUK Energy Programme (Grant No. EP/T012250/1). The first author has worked under a $\mathrm{PhD}$ grant financed by ENEA.

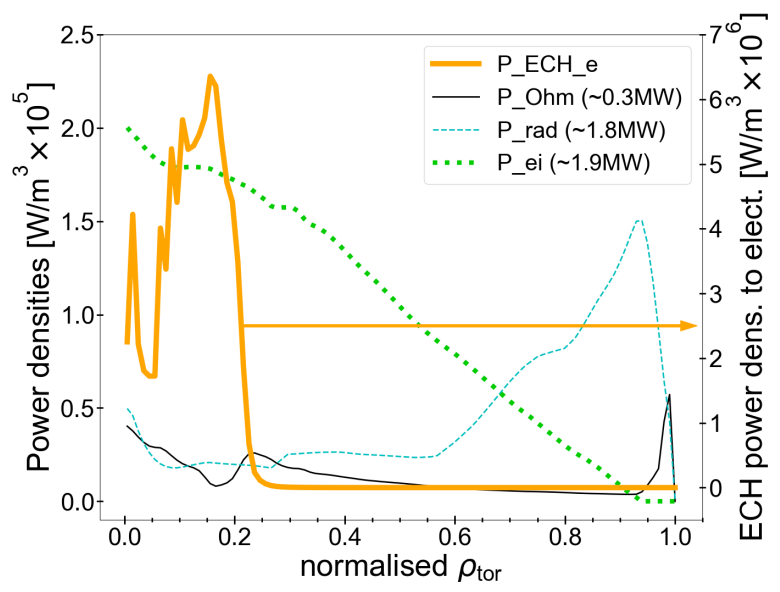

(a)

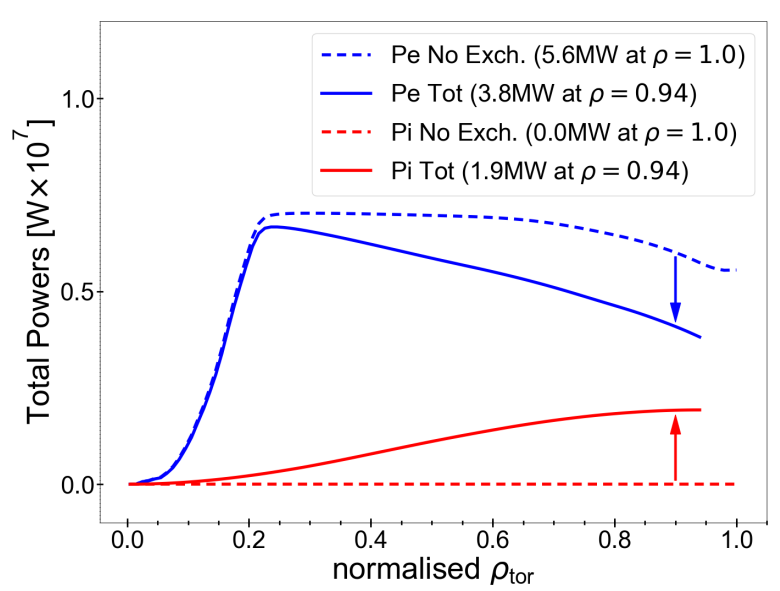

(b)

Figure 23: (a) Radial profiles of power densities in the day-0 scenario: ECRH power deposited to electrons $P_{\mathrm{ECH}}$, Ohmic power $P_{\mathrm{Ohm}}$, radiative power $P_{\mathrm{rad}}$, and thermal exchange power between electrons and ions $P_{e i}$. (b) Radial profiles of electron and ion total powers including or not including the thermal exchange power between species in the day-0 scenario. 


\section{References}

[1] Fusion Electricity - A roadmap to the realisation of fusion energy (2012), https://www.euro-fusion.org/fileadmin/ user_upload/Archive/wp-content/uploads/2013/01/JG12. 356-web.pdf

[2] European Research Roadmap to the Realisation of Fusion Energy, https://www.euro-fusion.org/fileadmin/user upload/EUROfusion/Documents/2018_Research_roadmap_ long_version_01.pdf

[3] https://www.iter.org/

[4] https://www.euro-fusion.org/programme/demo/

[5] G. Granucci et al Fusion Engineering and Design 122 (2017), 349-355

[6] Martone, R. Albanese, F. Crisanti, P. Martin, and A. Pizzuto (ed.), "Divertor Tokamak Test Facility: Interim Design Report", ISBN: 978-88-8286-378-4, April 2019, "Green Book", https://www.dtt-dms.enea.it/share/ s/avvglhVQT2aSkSgV9vuEtw

[7] https://www.dtt-project.enea.it/

[8] A. Pizzuto et al 2010 Nucl. Fusion 50095005

[9] D.M. Thomas 2006 Plasma Phys. Control. Fusion 48 A183

[10] ITER Organization 2018 ITER Research Plan within the Staged Approach (Level III - Provisional Version), report ITR18-003

[11] B. Baiocchi and P. Mantica, "Physics-based transport modelling of main species particle and energy transport in the core plasma of ITER reference scenarios during stationary phases and post-pellet transients", third report

[12] A.S. Kukushkin et al 2003 Nucl. Fusion 43716

[13] R. Wenninger et al 2017 Nucl. Fusion 57016011

[14] M. Greenwald 2002 Plasma Phys. Control. Fusion 44 R27

[15] M. Romanelli, Plasma and Fus. Research 9, 3403023 (2014)

[16] G. V. Pereverzev and P. N. Yushmanov, ASTRA automated system for transport analysis in a tokamak IPP Report 5/98, Max-Planck-Institut für Plasmaphysik (2002)

[17] S Saarelma et al 2018 Plasma Phys. Control. Fusion 60014042

[18] P.B. Snyder et al 2011 Nucl. Fusion 51103016

[19] E. Stefanikova et al 2018 Nucl. Fusion 58056010

[20] L. Frassinetti et al 2021 Nucl. Fusion 61016001

[21] G. M. Staebler et al 2016 Physics of Plasmas 236062518

[22] J. E. Kinsey et al 2008 Physics of Plasmas 155055908

[23] G. M. Staebler et al 2007 Physics of Plasmas 145055909

[24] G. M. Staebler et al 2005 Physics of Plasmas 1210102508

[25] J. Citrin et al 2017 Plasma Phys. Control. Fusion 59124005

[26] C. Bourdelle et al 2016 Plasma Phys. Control. Fusion 58014036

[27] P. Mantica et al 2020 Plasma Phys. Control. Fusion 62014021

[28] S. Mordijck et al 2020 Nucl. Fusion 60066019

[29] B.A. Grierson et al 2018 Phys. Plasmas 25022509

[30] E.J. Doyle et al, FEC 2012, IAEA Fusion Energy Conference EX/P3-26

[31] C. Angioni et al 2019 Nucl. Fusion 59106007

[32] A. Ho et al 2019 Nucl. Fusion 59056007

[33] M. Marin et al 2020 Nucl. Fusion 60046007

[34] M. Marin et al 2021 Nucl. Fusion 61036042

[35] F.J. Casson et al 2020 Nucl. Fusion 60066029

[36] C.K. Kiefer et al 2021 Nucl. Fusion 61066035
[37] G. M. Staebler et al 2021 Plasma Phys. Control. Fusion 63 015013

[38] C. Stephens et al, to be submitted to Physics of Plasmas

[39] K. L. van de Plassche 2020 Physics of Plasma 272022310

[40] M. Romanelli and M. Ottaviani 1998 Plasma Phys. Control. Fusion 401767

[41] W. A. Houlberg et al 1997 Physics of Plasmas 49 3230-3242

[42] A. Peeters et al 2009 Physics of Plasmas 166062311

[43] A.G. Peeters et al 2011 Nucl. Fusion 51094027

[44] L. Taroni, 21st EPS Conf. Contr Fus Plas Phys - Montpellier 102 I (1994)

[45] G. Cenacchi and A. Taroni 1988 ENEA-RT-TIB 88-5

[46] A.A. Ivanov, 32nd EPS Conf. on Plasma Physics - Terragona 29C (ECA), P-5.063 (2005)

[47] R. Albanese, R. Ambrosino and M. Mattei 2015 Fusion Engineering and Design 96-97 664 - 667

[48] D. Farina 2007 Fusion Science and Technology 522 154-160

[49] C.D. Challis et al 1989 Nucl. Fusion 29563

[50] P. Agostinetti et al 2019 Fusion Engineering and Design 146 A 441-446

[51] L.-G. Eriksson et al 1993 Nucl. Fusion 331037

[52] T. Hellsten and L.-G. Eriksson 1989 Nucl. Fusion 292165

[53] T. Hellsten and L. Villard 1988 Nucl. Fusion 28285

[54] T. Görler et al 2011 Journal of Computational Physics 23018 7053-7071

[55] http://www.genecode.org/

[56] F. Romanelli and F. Zonca 1993 Physics of Fluids B: Plasma Physics 511 4081-4089

[57] F. Romanelli 1989 Physics of Fluids B: Plasma Physics 15 1018-1025

[58] M. Beurskens et al, IAEA FEC 2020, IAEA-CN-EX/6-3

[59] F. Jenko et al 2001 Physics of Plasmas 89 4096-4104

[60] A. Di Siena et al 2018 Nucl. Fusion 58054002

[61] A. Di Siena et al 2019 Nucl. Fusion 59124001

[62] A.M. Garofalo et al 2014 Fusion Engineering and Design 897 876-881

[63] P.B. Snyder et al 2011 Nucl. Fusion 51103016

[64] M. G. Dunne et al 2017 Plasma Phys. Control. Fusion 59 025010

[65] L. Frassinetti et al 2019 Nucl. Fusion 59076038

[66] U A Sheikh et al 2019 Plasma Phys. Control. Fusion 61014002

[67] H.Q. Wang et al 2018 Nuclear Fusion 58096014

[68] L. Frassinetti, EX/2-2 28th IAEA Fusion Energy Conference (FEC 2020)

[69] S. Tamor 1981 J. Comput. Phys. 40104

[70] L. Balbinot et al 2021 Nuclear Materials and Energy 27100952

[71] H. Bufferand et al 2013 Journal of Nuclear Materials 438 S445$\mathrm{S} 448$

[72] H. Bufferand et al 2015 Nuclear Fusion 55053025

[73] L.L. Lao et al 1985 Nucl. Fusion 251611

[74] M. Brix et al 2008 Rev. Sci. Instrum. 79 10F325

[75] R.L. Miller 1998 Phys. Plasmas 5973

[76] A. Mariani 2018 Phys. Plasmas 25012313

[77] A. Mariani 2019 Phys. Plasmas 61064005

[78] F. Jenko et al 2005 Plasma Phys. Control. Fusion 47 B195

[79] G. Spizzo et al, "Collisionless losses of fast ions in the Divertor Tokamak Test due to toroidal field ripple", submitted to Nucl. Fusion (NF-104519.R1)

[80] Liu Chen and Fulvio Zonca 2016 Rev. Mod. Phys. 88, 015008 